\title{
Determinants for efficient editing with cas9-mediated recombineering in Escherichia coli
}

Choudhury, Alaksh; Fankhauser, Reilly G.; Freed, Emily F.; Oh, Eun Joong; Morgenthaler, Andrew B.; Bassalo, Marcelo C.; Copley, Shelley D.; Kaar, Joel L.; Gill, Ryan T.

\section{Published in:}

ACS Synthetic Biology

Link to article, DOI:

10.1021/acssynbio.9b00440

Publication date:

2020

Document Version

Peer reviewed version

Link back to DTU Orbit

Citation $(A P A)$ :

Choudhury, A., Fankhauser, R. G., Freed, E. F., Oh, E. J., Morgenthaler, A. B., Bassalo, M. C., Copley, S. D., Kaar, J. L., \& Gill, R. T. (2020). Determinants for efficient editing with cas9-mediated recombineering in Escherichia coli. ACS Synthetic Biology, 9(5), 1083-1099. https://doi.org/10.1021/acssynbio.9b00440

\section{General rights}

Copyright and moral rights for the publications made accessible in the public portal are retained by the authors and/or other copyright owners and it is a condition of accessing publications that users recognise and abide by the legal requirements associated with these rights.

- Users may download and print one copy of any publication from the public portal for the purpose of private study or research.

- You may not further distribute the material or use it for any profit-making activity or commercial gain

- You may freely distribute the URL identifying the publication in the public portal 


\section{Determinants for efficient editing with Cas9-mediated recombineering in Escherichia coli}

Alaksh Choudhury ${ }^{1,5}$, Reilly G. Fankhauser ${ }^{2}$, Emily F. Freed ${ }^{2}$, Eun Joong Oh ${ }^{1,2}$, Andrew B. Morgenthaler ${ }^{3}$, Marcelo C. Bassalo ${ }^{3}$, Shelley D. Copley ${ }^{3}$, Joel L. Kaar ${ }^{1}$, Ryan T. Gill1,2,4*

1. Department of Chemical and Biological Engineering, University of Colorado, Boulder, USA, 80309.

2. Renewable \& Sustainable Energy Institute, University of Colorado, Boulder, USA, 80303.

3. Department of Molecular, Cellular and Developmental Biology, University of Colorado, Boulder, USA, 80309.

4. Novo Nordisk Foundation Center for Biosustainability, Danish Technical University, Copenhagen, Denmark.

5. IAME, UMR 1137, INSERM, Universités Paris Diderot et Paris Nord, 75018 Paris France

* Corresponding Author

\section{Correspondence}

Ryan T. Gill: rtg@biosustain.dtu.dk

\section{Keywords}

CRISPR-Cas, Genome editing, Off-target activity, Recombineering, Repair fidelity 


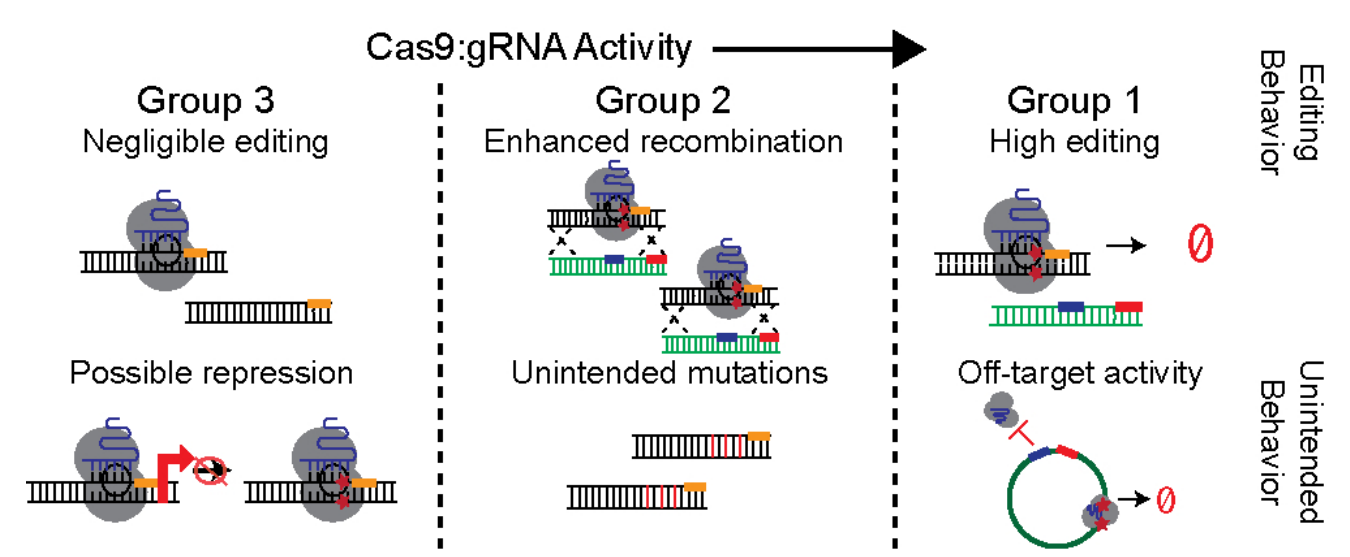

Table of contents figure 


\begin{abstract}
In E. coli, editing efficiency with Cas9-mediated recombineering varies across targets due to differences in the level of Cas9:gRNA-mediated DNA double-strand break (DSB)-induced cell death. We found that editing efficiency with the same gRNA and repair template can also change with target position, cas 9 promoter strength, and growth conditions. Incomplete editing, off-target activity, non-targeted mutations, and failure to cleave target DNA even if Cas9 is bound also compromise editing efficiency. These effects on editing efficiency were gRNA-specific. We propose that differences in the efficiency of Cas9:gRNA-mediated DNA DSBs, as well as possible differences in binding of Cas9:gRNA complexes to their target sites, account for the observed variations in editing efficiency between gRNAs. We show that editing behavior using the same gRNA can be modified by mutating the gRNA spacer, which changes the DNA DSB activity. Finally, we discuss how variable editing with different gRNAs could limit highthroughput applications and provide strategies to overcome these limitations.
\end{abstract}


In the S. pyogenes type II CRISPR system, a guide RNA (gRNA) directs Cas9 to introduce a DNA double-strand break (DSB) by complementarity to a target DNA sequence adjacent to a PAM site (5'-NGG3') ${ }^{1}$. In bacteria such as E. coli, Cas9:gRNA-mediated DSBs induce cell death due to a lack of adequate repair pathways ${ }^{2,3}$ (Fig 1A). Cas9:gRNA-induced cell death can select for cells with mutations in the PAM or the "seed" region of the spacer (the 8-10 nucleotides proximal to the PAM) that prevent Cas9 binding and thus DSBs ${ }^{2}$. In E. coli, such mutations are often introduced by phage-based recombination machinery such as the lambda Red proteins ${ }^{2,4}$. The PAM-inactivating mutation can be coupled to point mutations, deletions, or insertions in a homology repair template (HRT). Elimination of unedited cells by Cas9:gRNAcleavage increases the efficiency of genome editing ${ }^{2}$ (Fig 1A).

The broad potential of this technology has been realized with multiplexed and high-throughput platforms. The gRNA can be placed in cis with a HRT on a small 200-230 bp editing cassette ${ }^{5}$ (Fig 1A). Tens to hundreds of thousands of such editing cassettes can be synthesized on microarray chips and subsequently used to develop genome-wide libraries ${ }^{5}$. Oligomers with multiple editing cassettes or recursive introduction of cassettes can be used to make combinatorial libraries ${ }^{6,7}$. Such technologies have enabled fitness mapping and multiplexed engineering at the gene, pathway and genome levels ${ }^{5-9}$.

Despite its tremendous potential, Cas9-mediated recombineering remains difficult to control and exploit reliably. In $E$. coli, editing efficiency varies significantly between targets ${ }^{5,10}$. This variation has been attributed to variable DSB-induced cell death caused by gRNAs with different targets ${ }^{11,12}$. gRNAs that cause negligible DSB-induced cell death are unable to select for cells with the PAM/seed mutation(s) and consequently editing efficiency is decreased ${ }^{10}$. Problems due to inconsistent Cas9:gRNA activity can be exacerbated in high-throughput and multiplexed applications. In E. coli, 10-65\% of randomly screened gRNAs fail to induce cell death ${ }^{10-12}$. These non-selective gRNAs compromise the coverage of mutations in high-throughput libraries. The editing cassettes, each with a unique gRNA and HRT, are also used as barcodes to track mutations ${ }^{5}$. Therefore, non-edited cells with non-functional editing cassettes can be 
falsely tracked as indicating beneficial mutations in the genome and increase subsequent validation efforts and costs ${ }^{9}$.

It has been proposed that the challenges with inconsistent editing efficiency might be overcome by predicting gRNAs that efficiently cause DSBs. However, even using prescreened gRNAs that cause high Cas9:gRNA-induced cell death, a recent study found that editing efficiency varied between $0-100 \%{ }^{10}$. Thus, an improved understanding of the system is necessary to improve editing in single and highthroughput applications of Cas9-mediated recombineering in E. coli. In mammalian cells, factors such as Cas9 expression level, target position, off-target activity, experimental conditions, and gRNA design have been found to impact Cas9-mediated editing ${ }^{13}$. However, the impacts of such parameters on Cas9-mediated recombineering in bacteria are poorly understood. The goal of this study was to improve the understanding of Cas9-mediated recombineering in E. coli. Specifically, we determined the variation in editing efficiency with 35 cassettes with unique gRNAs and HRTs. For a subset of these cassettes, we studied the impact of target position, Cas9 expression level, gRNA spacer sequence, and growth conditions on editing efficiency.

\section{Results}

\section{Efficient gRNA-mediated cell death does not ensure high editing efficiency}

We first evaluated the variation in Cas9:gRNA-induced cell death using 35 gRNAs ( Supplementary Table 1). Of these, 16 targeted galK, which encodes galactokinase, and 19 targeted other genes across the genome. To quantify Cas9:gRNA-induced cell death, we calculated the fold reduction in colony-forming units (CFUs) after transforming a plasmid encoding either a targeting gRNA or a nontargeting gRNA into cells expressing Cas9 ${ }^{10}$ (Methods). The fold-reduction in CFUs varied over four orders of magnitude between gRNAs (Fig 1B).

We next evaluated the level of Cas9:gRNA-induced cell death required to enable high editing efficiency by selecting against cells without targeted genomic mutations. We constructed plasmids with an editing cassette encoding each gRNA in cis with a 150 bp HRT containing a synonymous PAM/seed 
mutation and a targeted point mutation(s) using previously optimized design rules ${ }^{5}$ (Fig $\mathbf{1 A}$ and Supplementary Table 1). Plasmids with the editing cassettes were transformed into cells with active Cas9 and lambda Red recombination proteins (Methods). Editing efficiency was measured by Sanger sequencing the target genomic locus from 20 colonies. Editing efficiency increased with an increase in the level of Cas9:gRNA-induced cell death measured for the gRNA of each cassette in the absence of a HRT (Fig 1B). Most gRNAs that resulted in efficient killing of unedited cells (i.e. those that showed greater than a 1000fold reduction in CFUs after transformation when compared to the CFUs upon transforming a non-targeting gRNA in the absence of a HRT) enabled nearly 100\% editing efficiency (Fig 1B). We categorized the 15 gRNAs above this threshold into Group 1. Nine gRNAs that caused negligible Cas9:gRNA-induced cell death in the absence of a HRT (with a p-value $>0.05$ compared to a non-targeting gRNA) were categorized into Group 3. The remaining gRNAs were categorized into Group 2. Some Group 1 gRNAs with high Cas9:gRNA-induced cell death had low editing efficiency. This suggested that high Cas9:gRNA-induced cell death does not always ensure high editing efficiency.

Cas9:gRNA-induced cell death in E. coli in the absence of a HRT and lambda Red recombination, as we measured in the experiment depicted in Fig 1B, is a proxy for Cas9:gRNA-mediated DNA DSB activity ${ }^{2,14}$. Therefore, we infer that the Cas9:gRNA-mediated DNA DSB activity increases from Group 3 to Group 1. Cas9:gRNA-mediated DSB activity is controlled primarily by interactions between the $20 \mathrm{bp}$ spacer, the target sequence, and Cas $9{ }^{15-17}$. We hypothesize that the variation in binding affinity between the spacer and the target sequence may be responsible for the large variation observed in Cas9:gRNAinduced cell death $($ Fig 1B).

Genome editing could occur by recombination between the genome and the HRT either before or after Cas9:gRNA-induced DSB formation. We observed a low level of edited CFUs $\left(\sim 10^{4}\right.$ edited out of $10^{6}$ total cells) in the presence of a HRT even with a non-targeting gRNA, which cannot cause DNA DSBs, suggesting that recombination catalyzed by the lambda Red recombination enzymes occurs independent of Cas9:gRNA-induced DSBs (Fig 1C). This is expected, as Cas9:gRNA-induced DSBs are primarily used as 


\section{Apparent editing efficiency for the same cassette changes with target genome position}

We next explored other parameters that could impact editing efficiency using the MacConkey agar screen to measure editing efficiency in galK. On MacConkey agar, colonies with inactive GalK are white, and colonies with active GalK are red ${ }^{21}$ (Fig 2A). We used nine editing cassettes to introduce a premature stop codon into galK. Of these, four had Group 1, two had Group 2, and three had Group 3 gRNAs (Supplementary Table 1). The percentage of white colonies is a measure of "apparent" editing efficiency, 
which may differ from actual editing efficiency because white colonies can arise by other mechanisms, which will be discussed below.

In E. coli, Cas9:gRNA binding, and lambda Red-mediated recombination, all of which contribute to editing efficiency, vary with genome position ${ }^{22-24}$. To test if editing efficiency varies with the target position, we deleted galK from its native operon ${ }^{21}$, then constructed strains with galK integrated at seven different genomic positions (Fig 2B and Supplementary Figure 2). For editing cassettes with Group 1 gRNAs, the percentage of white colonies remained high for all positions (Fig 2C). For editing cassettes with Group 2 gRNAs, the percentage of white colonies increased with increasing distance from the terminus (Fig 2C). Group 3 did not have a clear position-dependent trend, but the percentage of white colonies was highest furthest from the terminus of replication (Fig 2C). Therefore, the genomic position of the target is an important determinant of editing efficiency, particularly for gRNAs that cause low Cas9:gRNA-induced cell death.

\section{Apparent editing efficiency for the same cassette depends on cas9 expression level}

In $E$. coli, a single Cas9:gRNA complex can take up to six hours to find its target ${ }^{24}$. We posited that increasing the concentration of Cas9:gRNA complexes could increase the rate of target binding and thereby increase DSB activity. We kept gRNA expression constant by expressing it under the strong J23119 promoter in all cases, but swapped the inducible araC promoter that controlled cas 9 in the initial experiments with three previously characterized constitutive promoters: pro1 (weak), proC (medium), and proD (strong) ${ }^{25}$. The concentration of Cas9 increased across the pro1, proC and proD promoters (Supplementary Figure 3). The araC promoter resulted in a high concentration of Cas9 after induction, but negligible amounts of Cas9 when the promoter is not induced (Supplementary Figure 3). Since the araC promoter is not induced on the MacConkey plates (the arabinose interferes with the MacConkey assay), whereas the pro1, proC, and proD promoters are active on the MacConkey plates, it is somewhat difficult to compare $\mathrm{Cas} 9$ concentration with the araC promoter to Cas 9 concentration with the pro1, proC, and proD promoters. Nonetheless, we observe clear promoter-dependent trends in editing efficiency. We observed a significant increase in the percentage of white colonies for the two Group 2 cassettes and two 


\section{Introduction of targeted mutations using Group 1 gRNAs is reduced due to incomplete editing and deletions in the editing plasmid}

For Group 1 gRNAs, actual editing efficiency for galK integrated at three different sites in the genome was high $(80.5+/-1.5 \%$ and $90.5+/-2.2 \%$ cells had all the desired mutations with cassettes 1 and 2, respectively), similar to the percentage of white colonies (Fig 3A). The number of CFUs after transforming the plasmids encoding the editing cassettes (Fig 3A) was low $\left(10^{4}-10^{5}\right.$ CFUs per transformation) across all genomic positions compared to the number after transformation with plasmids encoding a non-targeting gRNA $\left(10^{6}-10^{7}\right.$ CFUs per transformation) (Fig 1C). This suggested that Group 1 gRNAs likely maintained high and consistent Cas9:gRNA-induced cell death across all genomic positions, allowing efficient elimination of non-edited cells and therefore high editing efficiency.

Due to the high Cas9:gRNA-induced cell death in Group 1, escape from Cas9:gRNA activity would be necessary for cell survival. We evaluated the escape mechanisms in the cells without all the desired 
mutations with Group 1 cassettes ( $\sim 20 \%$ and $\sim 10 \%$ of all cells for cassettes 1 and 2 , respectively). In Cassette 1, the point mutations in the PAM and target site were separated by 18 bases. After editing with Cassette 1 , the deep-sequencing data showed that $\sim 92 \%$ of the reads had the PAM mutation and $\sim 81 \%$ of the reads had both the PAM and target mutation (Fig 3B, top). A subpopulation of cells $(\sim 10 \%)$ harbored only the PAM mutation for cassette 1. The PAM mutation alone should be sufficient to prevent Cas9:gRNA-mediated DSB activity; the targeted mutation itself is not necessary. In each of these cases, we observed that the bases at each position were either the wild-type residue or the mutation introduced by the repair template. Therefore, the reduction in frequency could be due to repair of the mismatches. The corrections are likely made by the methyl-directed mismatch repair (MMR) machinery. Lambda Red recombineering is proposed to occur via a single stranded intermediate that invades the lagging strand during replication. When mismatches in the template are encountered while resolving the invading strand, these mismatches are repaired by the MMR machinery which repairs mutations introduced by lambda Red recombination using single-stranded HRTs ${ }^{27,28}$. Cassette 2 had several target mutations in the seed region but no PAM mutation. It has been proposed that several consecutive mismatches in the genome may allow evasion of MMR. Although the actual editing efficiency with Cassette 2 was $10 \%$ higher than with Cassette 1, the base-change frequency of the mutations within the seed region still varied by over $8 \%$ (Fig $\mathbf{3 B}$, bottom). Therefore, editing was incomplete, irrespective of the HRT design. However, all partially edited cells for both cassettes harbored either a PAM mutation or at least 1 mutation in the seed region that allowed escape from Cas9:gRNA-mediated DSB activity.

Successful editing is the desired mechanism for escape, but clearly some unedited cells manage to escape as well. Nine percent and four percent of all the deep-sequencing reads did not have any PAM or seed mutation after editing with Cassettes 1 and 2, respectively (Fig 3B). There are three likely escape routes for unedited cells: 1) deletion of the target gene (galK);2) mutations in the target gene; or 3) mutations in the editing plasmid. We did not observe any deletions of galK (Supplementary Figure 4A) or mutations in galK (Supplementary Figure 4B). Upon sequencing the gRNA encoding plasmid we found that in $75 \%$ of these cases, escape could be attributed to random deletions in the editing plasmid that 


\section{Off-target DSB activity reduces editing efficiency for Group 1 gRNAs}

The number of CFUs after transformation with plasmids carrying cassettes using a Group 1 gRNA decreased with an increase in cas 9 promoter strength (Fig 3C). The decrease in the number of CFUs was not due to any increase in toxicity due to increased $\operatorname{cas} 9$ expression because we did not observe any significant change in the number of CFUs after transformation with null-plasmids without any gRNA (maintained at $\sim 10^{\wedge} 6$ CFUs per transformation, $\mathrm{p}>0.05$ student's t-test). Therefore, the decrease in the total CFUs after transformation could be due to increased Cas9:gRNA induced cell death. In theory, an increase in Cas9:gRNA-induced cell death should increase editing efficiency due to the elimination of unedited cells. Contrary to this expectation, actual editing efficiency decreased with an increase in $\operatorname{cas} 9$ promoter strength (Fig 3C). Additionally, the fraction of cells with incomplete edits i.e., those with just synonymous PAM/SEED mutations that could provide escape from Cas9:gRNA induced cell death also decreased (Supplementary Figure 6A). This suggested that the percentage of cells that could have escaped using the dominant plasmid deletion mechanism may have increased (Supplementary Figure 6B). We observed that the number of cells per transformation that survived due to escape remained the same (Supplementary Figure 7). Since the number of edited cells decreased, likely due to off-target toxicity, and the number of escapees remained the same, the overall editing efficiency decreased.

These observations could be due to an increase in off-target activity of Cas9. Increased Cas9 expression has previously been shown to increase off-target binding of Cas9 in E. coli ${ }^{29}$. Cas9 can cause off-target DSBs in loci that have a PAM site in proximity to sequences with significant similarity to the N20 gRNA spacer ${ }^{2,30}$. However, the number of unedited cells in the population that escape due to deletion of the gRNA in the editing plasmid would be unaffected by Cas9 expression level, as the gRNA is required for activation of Cas9 for endonuclease activity. Therefore, we hypothesized that off-target DSB formation killed properly edited cells when Cas9 was overly abundant (Fig 3D). 
To test the impact of off-target DSBs on editing efficiency, we compared actual editing efficiency in the wild-type strain and a strain lacking $d g o K$, which contains a possible off-target site for Group 1 Cassette 1 (Fig 3D). With the strongest $\operatorname{cas} 9$ promoter (proD), actual editing efficiency decreased by $48+/-$ $3 \%$ in the wild-type strain but remained unchanged for the $\Delta d g o K$ strain (Fig 3E and Supplementary

Figure 8A). There was also a significant increase in the number of CFUs after transformation with plasmid carrying the editing cassette in the $\Delta d g o K$ strain compared to the wild-type strain $(\mathrm{P}<0.05)$ (Supplementary Figure 8B), suggesting a decrease in Cas9:gRNA-induced cell death due to lack of the off-target site. We conclude that off-target sites for Group 1 gRNAs reduce editing efficiency possibly because they confer both high on-target DSB activity and substantial off-target activity.

\section{Actual editing efficiency with Group 2 gRNAs changes with target position and cas9 promoter strength}

Group 2 gRNAs cause lower Cas9:gRNA-induced cell death in the absence of a HRT than Group 1 gRNAs. Therefore less-efficient selection against non-edited cells after editing using a Group 2 gRNA leads to lower editing efficiency (Fig 1B). For Group 2 Cassette 4, we observed a 34.0 +/- 1.2-fold increase in actual editing efficiency furthest from ter compared to actual editing efficiency at a position close to ter (Fig 4A, left). The increase in actual editing efficiency could be explained by an increase in Cas9:gRNAinduced cell death with increasing distance from ter (Supplementary Figure 9A). The position-dependent effects in Cas9:gRNA activity may be due to differences in Cas9:gRNA binding with position, which has been demonstrated using in vivo binding experiments in E. coli ${ }^{24}$.

We also observed a $5.4 \pm 0.05$-fold increase in actual editing efficiency with the pro1 cas 9 promoter compared to the araC $\operatorname{cas} 9$ promoter for Group 2 cassette 4 (Fig 4A, right). However, the editing efficiency decreased from $75.2 \%$ to $64.1 \%$ when $\operatorname{cas} 9$ was expressed using the proD promoter compared to expression under the prol promoter. The increase in editing efficiency was accompanied by an increase in Cas9:gRNAinduced cell death. This suggested that the Cas9:gRNA-induced cell death in Group 2 cassette 4 may be 
To evaluate the role of error-prone repair of DSBs, we transformed cells expressing Cas9 with plasmids encoding gRNAs without a HRT. We observed a high mutation frequency in the spacer region of

the DNA target (Fig 4D and Supplementary Figure 11A), as expected since mutations introduced by

\begin{abstract}
limited by the kinetics of DNA DSBs. The dependence on promoter strength may be due to improved DNA DSB kinetics with an increase in the number of Cas9:gRNA complexes which in turn improved the killing of non-edited cells. We discuss the decrease in editing efficiency with the strongest promoter in the next section.
\end{abstract}

A significant increase in actual editing efficiency with increasing distance from ter and with an increase in $\operatorname{cas} 9$ promoter strength from araC to pro1 was also observed for Group 2 Cassette 5 (Fig 4B) (P $<0.05$, Student's t-test). Interestingly, the number of CFUs remained unchanged after transformation with plasmids encoding Cassette 5 with or without the HRT across positions and $\operatorname{cas} 9$ promoters (p-value $>0.1)$ (Fig 4B and Supplementary Figure 9B). Therefore, in this case, the increase in editing efficiency can be attributed primarily to an increase in recombination with the HRT and not to increased DSB-induced killing of unedited cells.

\title{
Unintended mutations increased significantly with Group 2 gRNAs
}

We observed a significant number of mutations in the DNA spacer region that were not targeted for editing with both Group 2 cassettes at all levels of Cas9 expression (Fig 4C and Supplementary Figure 10A). These observed mutations were not due to MMR because changes in mutation frequency due to MMR occurred only in the residues targeted for recombination and the bases were corrected back to the wild-type residue as observed above (Figure 3B). However, in the case of group 2 gRNAs, the mutations occurred in residues other than those targeted by recombination and were random. These unintended mutations may be caused by error-prone polymerases induced by the SOS response to DNA damage ${ }^{31}$. Such polymerases localize at the DNA DSB site and introduce random mutations during the repair process 32. 
error-prone DSB repair of the Cas9:gRNA target can prevent subsequent Cas9:gRNA-mediated DSBs. The frequency of unintended mutations with Group 2 gRNAs increased significantly ( $p<0.05$, Student's t-test) with an increase in $\operatorname{cas} 9$ promoter strength (Supplementary Figure 10A). With the strongest proD promoter, a significant increase in the unintended mutations led to the observed decrease in editing efficiency. An increase in $\operatorname{cas} 9$ promoter strength may increase the frequency of DSBs, and increased DSB is positively correlated with the SOS response ${ }^{31}$. Further, induction of the lambda Red recombination system could impact the SOS response as the lambda Gam protein inhibits RecBCD endonuclease, which loads RecA onto resected DNA after the DSB and RecA binding induces the SOS response ${ }^{33}$. We repeated the previous experiment in cells by transforming the Group 2 gRNA into cells with lambda Red recombineering induced. The unintended mutations helped the cells escape high cas9:gRNA-induced cell death when a plasmid encoding Group 2 Cassette 4 gRNA was transformed into cells with strong cas 9 expression (using proD promoter) (Supplementary Figure 11B). We observed an order of magnitude decrease in the number of escapee colonies when the Group 2 Cassette 4 gRNA was transformed into cells with strong cas 9 expression with lambda Red proteins induced, suggesting a decrease in the frequency of unintended mutations (Supplementary Figure 11B). We also observed a slight decrease in the frequency of mutations when lambda Red enzymes are induced prior to the introduction of Group 2 gRNA plasmids without a HRT (Supplementary Figure 11C). This decrease in the frequency of unintended mutations, upon possible inhibition of SOS-response due to induction of the lambda Red Gam, further points to the role of response-mediated error-prone polymerases. Such increase in unintended mutations in response to Cas9:gRNA mediated DNA DSBs has not been reported previously in E. coli.

\section{Group 3 gRNAs cause binding of Cas9 but not DSB formation, dependent upon target position and cas9 promoter}

The percentage of white colonies approached $100 \%$ at positions farther from ter for all Group 3 cassettes and approached $100 \%$ with a change in the cas 9 promoter for Group 3 Cassettes 6 and 7 (Fig 5A). Surprisingly, actual editing efficiency as determined by sequencing galK in white colonies was significantly 

cas9 promoters (Fig 5A). It has been observed previously that inefficient gRNAs may cause a gene deletion resulting in mixed colonies with deleted and wild-type cells. We posited that the observed white colonies could be due to such mixed colonies. As discussed earlier, a shortcoming of deep-sequencing is that large deletions may not be captured. Therefore, we re-streaked several white colonies on MacConkey agar plates and observed a mixture of red and white colonies (Supplementary Figure 12A). Surprisingly, upon sequencing 20 white colonies, we did not observe any mutations or deletions. Loss of GalK function without any genomic mutations was also observed when Cas9-expressing cells were transformed with plasmids containing the Group 3 gRNA but no HRT (Supplementary Figure 13A).

Group 3 gRNAs cause negligible Cas9:gRNA-induced cell death in the absence of a HRT (Fig 1B), likely due to low DNA DSB activity. It appears that Group 3 gRNAs promote binding of Cas9, but not the conformational change that is required to induce a DNA DSB ${ }^{34,35}$. Therefore, the high percentage of white colonies with Group 3 gRNAs may be due to repression of galK transcription by bound Cas9. In order to test this hypothesis, we cloned two Group 3 gRNAs into plasmids with a temperature-sensitive origin of replication that are maintained at $30^{\circ} \mathrm{C}$ but can be cured at $37{ }^{\circ} \mathrm{C}{ }^{36}$. Colonies containing GalK inactivated by editing or unintended genomic mutations should remain white regardless of growth temperature, i.e., whether or not the gRNA is still present, as genome mutations are permanent. After transforming cells with plasmids carrying Group 3 gRNAs, the cells formed white colonies at $30^{\circ} \mathrm{C}$, but red colonies upon replating at $37^{\circ} \mathrm{C}$, conditions under which the plasmids expressing the gRNA were cured (Fig 5B). When we restreaked Group 3 cassette 6 at $30{ }^{\circ} \mathrm{C}$ after curing, it retained its red phenotype (Supplementary Figure 14). We also observed loss of GalK function when cells expressing dead Cas9 (dCas9), which is capable of repressing gene expression but not of causing DSBs, were transformed with Group 3 gRNAs (Supplementary Figure 13B). Additionally, while we observed a mix of red and white colonies when we restreaked white colonies formed using non temperature-curable plasmids at $37^{\circ} \mathrm{C}$, we observed only red colonies with the temperature curable backbone (Supplementary Figure 15). Finally, the Group 3 Cassette 8 gRNA, which had similar Cas9:gRNA induced cell death as Cassettes 6 and 7, did not demonstrate the 
repression phenotype (Supplementary Figure 16). Cassette 6 and Cassette 7 gRNA targeted the nontemplate strand, but Cassette $8 \mathrm{gRNA}$ targeted the template strand. It has been previously demonstrated that non-template strand-targeting gRNAs have significantly more effective repression compared to template strand targeting-gRNAs ${ }^{37}$. These data suggested that the observed loss of function of GalK with some Group 3 cassettes could be due to transcriptional repression rather than actual editing.

Interestingly, we observed a high percentage of white colonies further from ter compared to positions closer to ter with negligible increases in actual editing efficiency. This suggested that apart from an increase in Cas9 expression, a change in target position could also cause repression-like behavior. The increase in repression further from ter again suggested that Cas9:gRNA binding increased with an increase in the distance from ter. The altered repression-like behavior could also be due to a change in Cas9:gRNA binding with position in E. coli ${ }^{24}$.

\section{Editing behavior can be controlled by the gRNA spacer sequence}

We hypothesized that limited DSB activity of Cas9:gRNA complexes might explain the cas 9 promoter strength- and target position-dependent editing efficiency observed for Group 2 gRNAs (Fig 4) and the repression of transcription observed for Group 3 gRNAs (Fig 5). To test this hypothesis, we attempted to decrease the DSB activity of two Group 1 gRNAs by mutating the 4 th, 8 th and 12 th nucleotides of the gRNA spacer ${ }^{30}$ and performed editing using the same HRT (Fig 6A). When $\operatorname{cas}^{9}$ was expressed using the araC promoter, actual editing efficiency decreased dramatically for both Cassette 1 and Cassette 2 variants (Fig 6A). Expressing cas 9 using the proC promoter increased actual editing efficiency using the Mut-4 and Mut-12 variants of Cassette 1 by $6.1 \pm 1.0$ - and $15.0 \pm 1.0$-fold, respectively, compared to the actual editing efficiency when cas 9 was expressed using the $\operatorname{araC}$ promoter (Fig 6A). However, the actual editing efficiency for variants of Cassette 2 showed a different trend. Actual editing efficiency with the Mut-4 variant of Cassette 2 was similar to that with the wild-type gRNA, but actual editing efficiency for the Mut- 8 and Mut-12 variants was negligible when $\operatorname{cas} 9$ was expressed using either the araC promoter or proC promoter (Fig 6A). Interestingly, the percentage of white colonies for with the Mut-8 and Mut-12 


\section{Editing behavior changes with growth phase}

Reduced cell growth rate may affect editing as cell growth impacts lambda Red recombination. Additionally, reduced cell growth may also give Cas9 more time to dissociate from its target so that recombination proteins may also access the DSB site. We evaluated the impact of reduced cell growth rate on actual editing efficiency by (i) recovering the cells in M9 minimal medium rather than in rich medium (LB); and (ii) editing cells in early stationary phase (defined as $\mathrm{OD}_{600}=2.5$ ) using Cassette 1 from Group 
1, Cassette 5 from Group 2 and Cassette 6 from Group 3 and using the araC promoter for cas 9 expression. Slowing cell growth substantially improved editing efficiency for Group 2 and 3 gRNAs (Fig 7A). Editing in stationary phase increased editing efficiency by $15.6 \pm 1.7$-fold and 5.4 \pm 0.2 -fold with single Group 2 and Group 3 gRNAs, respectively (Fig 7A). The increase in actual editing efficiency occurred without significant change in total CFUs per transformation for Groups 2 and Groups 3, suggesting an increase in DSB-dependent recombination. With the Group 1 gRNA, editing efficiency remained high, but the number of CFUs after transformation increased significantly when editing was carried out in the stationary phase. As the replication machinery is proposed to interfere with Cas9:gRNA binding ${ }^{24}$, one possible explanation for the increase in editing efficiency could be that binding of the Cas9:gRNA complex to its genomic DNA target in E. coli increased due to the decreased cell growth rate.

\section{High editing efficiency with a mixture of editing cassettes requires complex control over several parameters}

We next evaluated the impact of variable editing behavior across cassettes on editing in multiplex using an equimolar mixture of one cassette from each group of gRNAs (Cassettes 1, 5 and 6) and a nontargeting gRNA at the wild-type position of galK, with cas 9 expressed using the araC promoter. The nontargeting gRNA represented non-functional gRNAs in high-throughput libraries introduced by errors in synthesis and cloning, which can comprise up to $30 \%$ of a library ${ }^{5}$. The total actual editing efficiency using the mixture of cassettes was only $1.1 \pm 0.04 \%$ (Fig 7B). Usually, plasmids encoding cassettes with high editing efficiency yield orders of magnitude fewer CFUs after editing than plasmids encoding non-targeting gRNAs and poorly editing Group 2 and Group 3 cassettes (Fig 1B). During editing with mixtures of cassettes, this significant bias in cell survival leads to low editing efficiency. Therefore, in order to increase high-throughput editing efficiency in E. coli, we need to improve both editing efficiency and overcome the bias in the number of CFUs after editing. Improved editing efficiency was achieved with single cassettes by editing in stationary phase (Fig 7A). Upon editing with a mixture of cassettes from each of the three groups and a non-targeting gRNA, the total actual editing efficiency increased by $11.0 \pm 0.9$-fold (Fig 7B). 


\section{Discussion}

Editing is an outcome of three processes: 1) cell death induced by DNA DSBs, 2) lambda Red recombination, and 3) escape. Initially we highlight that high editing occurs primarily due to elimination of unedited cells via Cas9:gRNA-induced cell death with a slight DSB-induced improvement in lambda Redmediated recombination (Figure 1). Therefore, the editing efficiency is primarily determined by Cas9:gRNA-induced cell death. Recently, Cui and Bikard suggested that Cas9:gRNA-induced cell death is caused only when the Cas9:gRNA complexes cleave all copies of the chromosome ${ }^{11}$; if sister chromatids are not available to allow repair of the cleaved chromosome using native homologous recombination, the cell dies. Therefore, Cas9:gRNA-induced cell death is determined by the kinetics of the DNA DSB activity of the Cas9:gRNA complex. DNA DSB kinetics are, in turn, controlled by the binding of the Cas9:gRNA complex to its target which depends on the interaction of the gRNA spacer sequence with the target DNA ${ }^{17}$. Several models are available to explain binding of the Cas9:gRNA complex to the target DNA ${ }^{13}$. One of these models, recently described by Farasat and Salis, may help explain some results across gRNA groups. According to this model, the binding energy of the Cas9: gRNA complex, which controls the DNA DSB activity, is determined by three parameters:

$$
\Delta \mathrm{G}_{\text {target }}=\Delta \mathrm{G}_{\mathrm{PAM}}+\Delta \Delta \mathrm{G}_{\text {Exchange }}+\Delta \Delta \mathrm{G}_{\text {Supercoiling }}
$$

Where $\Delta \mathrm{G}_{\text {target }}$ is the free energy of binding and depends on $\Delta \mathrm{G}_{\mathrm{PAM}}$, the energy released by the interaction between Cas9 and the PAM, $\Delta \Delta \mathrm{G}_{\text {Exchange, }}$, the energy required for the formation of the R-loop of the DNA:RNA:DNA complex when the DNA:RNA base pairing sequentially replaces the DNA:DNA base- 
pairing, and $\Delta \Delta \mathrm{G}_{\text {Supercoiling, }}$ the energy required to uncoil the DNA. The DNA DSB can only be induced once favorable binding is initiated ${ }^{39,40}$. In Group 1 the Cas9:gRNA-induced cell death and editing efficiency is high and consistent across positions (Figure 2). We posit that Group 1 gRNAs have strong binding likely due to a very low $\Delta \Delta \mathrm{G}_{\text {Exchange. }}$ Therefore, the kinetics of DNA DSB are favorable. Due to a low $\Delta \Delta \mathrm{G}_{\text {Exchange, }}$ the Cas9:gRNA activity is likely never limited by binding and remains consistent regardless of genome position (Figure 2 and 3). In Group 2, the kinetics of DNA DSB may be less favorable likely due to a lower $\Delta \Delta \mathrm{G}_{\text {exchange }}$ compared to Group 1 . Therefore, when we increase the cas 9 promoter strength, the DNA DSB kinetics increase and consequently, we observe improved editing. Finally, in Group 3, we suspect that the $\Delta \Delta \mathrm{G}_{\text {Exchange }}$ is likely the lowest and binding is not favorable for inducing DNA DSBs. Similar repression behavior has been demonstrated with gRNAs with shorter spacers which have unfavorable gRNA:DNA interactions ${ }^{41}$.

One of the limitations of our study is that we did not directly measure the binding of Cas9:gRNA to its target across different spacers. As an alternative, we performed editing after introducing mutations in Group 1 gRNAs that are proposed to reduce the binding affinity of the Cas9:gRNA complex to its target $17,42,43$. When we introduced mutations in the gRNAs of Group 1 cassettes, we were able to convert their behavior to Group 2-like $\operatorname{cas} 9$ promoter-dependent editing and Group 3-like repression behavior (Figure 6). These observations further support the hypothesis that the strength of Cas9:gRNA binding may be a main determinant of editing behavior using Cas9:gRNA mediated recombineering. A quantitative measurement of the binding the gRNAs across groups would be required to validate the correlation between editing and gRNA binding.

In some cases, we observed a change in editing with a change in genome position. With Group 2 cassette 4, the increase in editing with position occurred with an increase in the Cas9:gRNA induced cell death (Figure 4 and Supplementary Figure 8). The Cas9:gRNA induced cell death is solely dependent on the Cas9:gRNA-induced DNA DSB activity. It has been demonstrated that the binding of a Cas9:gRNA complex can change with the genomic position of its target in E. coli ${ }^{24}$. However, the trend for dependence on distance from ter was interesting. In our experimental setup, we targeted the different positions using 

the same with a change in the position on the genome, as the PAM and the spacer are the same. However, the $\Delta \Delta \mathrm{G}_{\text {Supercoiling }}$ likely changes due to a change in supercoiling of the chromosome. This is further corroborated by the observed increase in the repression-like behavior for all Group 3 gRNAs closer to the origin, as the repression would primarily depend on gRNA binding (Figure 5A). In previous studies, it has been observed that DNA negative supercoiling increases closer to ter ${ }^{44}$. However, these significant differences were observed in stationary phase cells. It may be possible that slight supercoiling differences occur during exponential phase, during which we conducted our studies, that may not be easily detectable. However, the role of supercoiling in bacterial editing needs to be further explored. Other factors, such as regional variation in chromosome copy number due to incomplete replication or position-dependent differences in gene expression, may also contribute to changes in editing efficiency with genome position. Further studies are required to understand the exact reason for the position dependence of Cas9:gRNAinduced DNA DSB activity.

Another interesting observation in our study was that while escape in non-edited cells in Group 1 was primarily by deletions in the gRNA expressing plasmid, escape in Group 2 occurred via increased nonspecific mutations within the target. One possible explanation for the difference in escape mechanisms could be due to differences in Cas9:gRNA DNA DSB kinetics between Groups 1 and 2. However, in the case of Group 2 cassette 4 with the stronger $\operatorname{cas} 9$ promoters pro 1 and proC, the Cas9:gRNA-induced cell death was similar to Group 1 gRNAs (Figure 3, 4 and Supplementary Figure 8). This suggested that the kinetics of Cas9:gRNA-induced cell death were similar in both cases. Another possibility for the variation in mutation frequency could be position dependent variation in native repair activity. The DNA DSB repair is controlled by RecA. Therefore, DNA DSB repair can vary significantly with distance from Chi sites. The regions targeted by Group 1 and Group 2 gRNA occur within a 400 bp region of galK. However, one of the Group 1 gRNAs in our study was closer to the Chi site than a Group 2 gRNA. While mutations are observed with the Group 2 gRNA, we do not observe any mutations in the Group 1 gRNA. Therefore, differences in repair activity due to variable distance from Chi site is unlikely be a determining factor for variable mutation 
frequency. Most likely, the difference in the frequency of unintended mutations with Group 2 gRNAs versus Group 1 gRNAs may be due to differences in the dissociation rates of the Cas9:gRNA complexes. Cas9:gRNA complexes can stay bound to the DNA even after inducing the DSB, and persistent binding is proposed to decrease access of repair proteins to the DNA DSB ${ }^{45,46}$. It has also been demonstrated that improved dislodging of the Cas9:gRNA complex from the target improves repair fidelity ${ }^{45,47}$. Based on our observations, we hypothesize that the Cas9:gRNA complexes with Group 2 gRNAs may dissociate from the target site faster than Cas9:gRNA complexes with Group 1 gRNAs, which would then allow error-prone repair proteins to access the DSB. In contrast, Cas9:gRNA complexes with Group 1 gRNAs may bind more tightly and not dissociate from the DSB. The relatively stronger binding and slower dissociation may preclude error-prone repair and thereby making mutations in the plasmid the primary escape mechanism.

Our hypothesis of dissociation-dependent increase in non-specific mutations is corroborated by the increase in non-specific mutations observed with Group 1 cassettes harboring a mutated gRNA. Since mutations in the gRNA likely weaken the binding of the Cas9:gRNA complex to its target, they may also increase its dissociation rate. We observed that mutations in Group 1 gRNAs that resulted in Group 2-like cas 9 promoter-dependent editing behavior also resulted in an increase in non-specific mutations. Such nonspecific mutations were not observed while editing using the non-mutated gRNA. However, the correlation between the increased mutation rates as an escape mechanism and dissociation rates needs experimental confirmation.

Another interesting observation in our study was the increase in editing efficiency with Group 2 cassette 5 with a change in cas 9 promoter (Fig 4B) despite the very low Cas9:gRNA-induced cell death in the absence of a HRT (Supplementary Figure 3B). The low Cas9:gRNA-induced cell death suggested that selection against unedited cells is poor for this cassette. This observation may also be explained by the relatively fast dissociation of Cas9:Group 2 gRNA complexes. We hypothesize that when this cassette does bind and cleave the target site, its relatively fast dissociation from the DSB (due to its weak affinity to the target) may allow access of both error-prone DNA polymerases and recombination proteins that would facilitate recombination with the HRT. An increase in DSB activity due to increased Cas9 expression would 
increase DSB-mediated recombination with the HRT and thereby increase actual editing efficiency (Fig 4B). Therefore, in contrast to Group 1 gRNAs for which high editing efficiency is predominantly an outcome of high selective pressure against unedited cells, the high editing efficiency for Group 2 cassette 5 achieved at high Cas9 levels may be due to a combination of DSB-independent recombination with the HRT and increased recombination with the HRT after a DSB even in the absence of strong selection against unedited cells. Our hypothesis of the dissociation-dependent increase in recombination was further corroborated when we mutated the spacer for a Group 1 cassette. We observed an increase in DSB-mediated recombination for a Group 1 cassette (Figure 6), as mutations in the gRNA spacer should reduce the binding affinity between the Cas9:gRNA complexes and their DNA targets. However, this hypothesis also requires experimental validation.

Previous experiments have measured Cas9:gRNA-induced cell death to reveal principles for design of gRNAs with high DSB activity ${ }^{14}$. However, we demonstrate that high DNA DSB may not be the only criterion for optimal editing. We may need to screen for several other parameters while editing including off-target activity, non-specific mutations and the possibility of repression (in the case of loss-of-function mutations and knockouts). Additionally, while gRNAs with high DNA DSB may be useful for genome editing of single targets, they appear to be suboptimal in multiplex applications. Our results suggest that multiplex applications may work best with gRNAs that show Group 2 behavior and therefore promote both DSB-dependent and DSB-independent mechanisms of recombination with the HRT to enable high actual editing efficiency and CFUs after transformation. Therefore, we need a better understanding of the design principles for Group 2-like gRNAs. Alternatively, multiplex editing might be improved by engineering Cas9 nucleases with increased dissociation rates after DNA DSB formation. However, understanding gRNA design would still be crucial to ensure consistent editing efficiency across cassettes. Other challenges with multiplexed libraries also need to be addressed. Partial editing (Fig 3), off-target DSB activity (Fig 3), unintended mutations (Fig 4), and binding of Cas9 without DSB formation (Fig 5) also interfere with targeted editing. These factors pose problems for use of editing cassettes as barcodes to estimate the fitness of cells with targeted mutations because the plasmid barcode does not necessarily correspond to the targeted 
edit in the genome ${ }^{5}$. Specifically, the error-prone polymerases, which usually localize at the DSB site for repair, may also increase the overall mutation rate in the cell and cause off-target mutations. Such off-target mutations may impact cell growth and could convolute the fitness estimates. We propose the following strategies to minimize these problems: 1) improve HRT design to avoid incomplete editing (Fig 3); 2) use engineered high-fidelity nucleases to prevent elimination of edited cells due to off-target DSB activity ${ }^{48}$; and 3) establish control over error-prone polymerases to prevent the introduction of unintended mutations.

Single and multiplexed editing protocols using Cas9-mediated recombineering have been developed in several bacterial hosts ${ }^{2,49}$. Cas9:gRNA binding to target sequences should be similar in most bacterial hosts and the mechanisms of RecA recombination and the SOS response that impact editing efficiency are highly conserved across bacteria ${ }^{31,50}$. Therefore, our findings based on studies in E. coli may be broadly significant across bacterial hosts.

\section{Materials and Methods}

\section{Design of editing constructs}

All editing was performed using S. pyogenes Cas9-mediated lambda recombineering. In order to design gRNAs, we identified the NGG PAM closest to the target site. A 20 bp sequence homologous to the sequence upstream of the PAM was used as the gRNA spacer. In our editing cassettes for point mutations, we designed 150 bp homology repair templates (HRTs) centered around the NGG PAM. This template consisted a synonymous mutation to mutate the G in the NGG PAM to provide immunity to subsequent cleavage by Cas9 after editing as well as targeted mutation(s) within $20 \mathrm{bp}$ of the synonymous PAM mutation. This repair homology template was placed in cis with the gRNA, which was expressed under control of the $\mathrm{J} 23119$ promoter, in a $230 \mathrm{bp}$ editing cassette that was ordered as a gblock from Eurofins.

For targeted deletions and insertions, the plasmid containing the designed gRNA was cotransformed with a linear HRT. For targeted deletion of $d g o K$, the HRT was ordered as a 200 bp g-block with $100 \mathrm{bp}$ homology to sequences upstream and downstream of the targeted gene (Appendix

\section{Supplementary Table 1).}


In order to construct strains with galK integrated at different genomic loci, we first deleted the galK gene from its operon in E. coli MG1655. Several bases towards the end of the galK gene are involved in the expression of subsequent genes in the galactokinase pathway. Warming et al. previously found that, in order to keep this pathway functional for the MacConkey agar screen, a portion of the galK gene needed to be retained after deletion. Consequently, we amplified the HRT for deletion of galK from the SW105 strain 51 using primers galk_100_for and galk_100_rev to include this functional region. For integration of galactokinase $\left(\right.$ galK) at different genomic loci (Fig 2B), we used the method described by Bassalo et al ${ }^{52}$. Each integration plasmid contained a $\sim 1200$ bp region homologous to the integration site centered around the PAM in a pBR322 backbone. The plasmid initially contained a uvGFP cassette with 600 bp sequences homologous to the upstream and downstream regions of the destination site. We used primers from Appendix Supplementary Table 2 to replace uvGFP with galK in the plasmids. galK flanked by 600 bp homology regions was subsequently amplified for genome integration. Integration was performed by cotransforming the gRNA with the linear HRT as described above. Proper integration was verified using PCR and subsequent Sanger sequencing.

\section{Cloning}

We used NEB Q5 polymerase to amplify all gblocks, integration cassettes, and plasmid backbones. PCR was performed with $2.5 \mu \mathrm{L}$ each of $10 \mu \mathrm{M}$ forward and reverse primers, $1 \mu \mathrm{L}$ of template $(\sim 1-10$ $\mathrm{ng} / \mu \mathrm{L}), 25 \mu \mathrm{L}$ of $2 \mathrm{X}$ NEB Q5 $2 \mathrm{X}$ Master Mix and $19 \mu \mathrm{L}$ of nuclease-free double distilled water under standard PCR conditions for HF Phusion polymerase $\left(98^{\circ} \mathrm{C}\right.$ for $30 \mathrm{~s}, 34$ cycles of $98^{\circ} \mathrm{C}$ for $15 \mathrm{~s}, \mathrm{~T}_{\mathrm{m}}{ }^{\circ} \mathrm{C}$ for $15 \mathrm{~s}$ and $72{ }^{\circ} \mathrm{C}$ for $15 \mathrm{~s}$ (length of amplicon), and one cycle of $72{ }^{\circ} \mathrm{C}$ for $5 \mathrm{~m}$ ). Primer $\mathrm{T}_{\mathrm{m}}$ values were calculated using the NEB $\mathrm{T}_{\mathrm{m}}$ calculator. For all plasmid backbones, the amplification was followed by a DpnI digestion reaction; $1 \mu \mathrm{L}$ of NEB DpnI was added to the PCR amplification reaction and the solution was incubated at $37^{\circ} \mathrm{C}$ for $1 \mathrm{hr}$. Amplicons were purified by gel extraction using the Qiagen gel extraction kit. The inserts were cloned into plasmids using Circular Polymerase Extension Cloning (CPEC) with 12.5 $\mu \mathrm{L}$ of an equimolar mixture of insert and backbone with at least $100 \mathrm{ng}$ of backbone, and $12.5 \mu \mathrm{L}$ of NEB 
2X HF Phusion Master Mix. PCR reactions were carried out at $98{ }^{\circ} \mathrm{C}$ for $30 \mathrm{~s}$, followed by 10 cycles of 98 ${ }^{\circ} \mathrm{C}$ for $10 \mathrm{~s}, 55{ }^{\circ} \mathrm{C}$ for $10 \mathrm{~s}, 72{ }^{\circ} \mathrm{C}$ for $90 \mathrm{~s}$, and then $72{ }^{\circ} \mathrm{C}$ for $120 \mathrm{~s}$ followed by a hold at $12{ }^{\circ} \mathrm{C}$ ). Ten $\mu \mathrm{L}$ of the CPEC reaction was transformed into competent cells by electroporation. The transformed cells were plated on LB and appropriate antibiotics as listed in the table.

\section{Measuring Cas9 Expression using ELISA}

Overnight cultures of MG1655, MG1655 + AraC Cas9 + pSIM5, MG1655 + Pro1 Cas9 + pSIM5, MG1655 +ProC Cas9 + pSIM5, and MG1655 + ProD Cas9 + pSIM5 were grown in biological triplicate and diluted 100 -fold in $50 \mathrm{~mL}$ of fresh $\mathrm{LB}$ media with $50 \mu \mathrm{g} / \mathrm{mL}$ kanamycin and $25 \mu \mathrm{g} / \mathrm{mL}$ chloramphenicol. The AraC Cas9 expression system was induced with filter sterilized $0.1 \%$ arabinose (VWR, TCA0515). The Pro1, ProC, and ProD expression systems express Cas9 constitutively. Cultures were grown to $\mathrm{OD}_{600} 0.4$ then temperature induced in a shaking water bath at $42{ }^{\circ} \mathrm{C}$ and $200 \mathrm{RPM}$ for 15 minutes then chilled on ice for 15 minutes. These conditions exactly replicate our standard recombineering protocol. Chilled cells were spun down at $5260 \mathrm{rcf}$ for 6 minutes and resuspended in $500 \mu \mathrm{L}$ of lysis buffer (50 mM Tris-HCL pH 7.5, 200mM NaCl, 5 mM DTT, 1 mM PMSF, 1X HALT protease inhibitor cocktail (ThermoFisher cat. No. 78429)). Resuspended cells were sonicated 10 x 10 seconds at 50\% power with 30 seconds chilling on ice between pulses. The soluble and insoluble fractions were separated by centrifugation at $13,000 \mathrm{rcf}$ at $4{ }^{\circ} \mathrm{C}$ for 30 minutes. Total protein yield in both fractions was quantified with the Pierce 660 nm Protein Assay Reagent (ThermoFisher Cat. No. 22660). Cas9 protein expression was quantified with the EpiQuik CRISPR/Cas9 Assay ELISA Kit (Colorimetric) (EpiGentek Cat. No. P-4060) following the manufacturer's protocol. A constant $1.5 \mu \mathrm{g}$ of total protein was loaded into the assay in triplicate for each expression construct for the soluble fraction and in duplicate for the insoluble fraction.

\section{Cas9-mediated Lambda red recombination and selection}


All editing was performed in E. coli MG1655 (a K12 strain). The lambda Red recombineering protocol was followed using cells that had been previously transformed with either the pX2cas 9 or proNX2cas9 plasmid and the pSIM5 lambda recombination plasmid ${ }^{53}$. Starter cultures of cells containing the pSim5 recombineering plasmid and the plasmid expressing $\operatorname{cas} 9$ were grown at $30^{\circ} \mathrm{C}$ overnight. The starter cultures were diluted 1:100 fold into 50-250 mL cultures and grown to mid-exponential phase $\left(\mathrm{OD}_{600}\right.$ of 0.35-0.4). The cells were incubated at $42{ }^{\circ} \mathrm{C}$ for 15 minutes to induce lambda Red recombination proteins and then cooled on ice for 15-20 minutes. Aliquots $(45 \mathrm{~mL})$ were subjected to centrifugation at $7500 \mathrm{x} g$ at $4{ }^{\circ} \mathrm{C}$ for 3 minutes. The supernatant was discarded, and the pellets were washed in $25 \mathrm{~mL}$ ice-cold sterilized double distilled water by resuspending and centrifuging at $7500 \mathrm{x} g$ at $4{ }^{\circ} \mathrm{C}$ for 3 minutes. Cells were washed thrice in prechilled $\left(4^{\circ} \mathrm{C}\right)$ water and once in prechilled $\left(4^{\circ} \mathrm{C}\right) 10 \%$ glycerol. Finally, the cells were resuspended in a 100 -fold lower volume of $10 \%$ glycerol than the culture volume. For editing, cells were transformed with either the editing plasmids or with the guide RNA and the linear HRT in $0.1 \mathrm{~mm}$ electroporation cuvettes. Transformed cells were allowed to recover for three hours in either LB or LB with $0.2 \%$ arabinose when the araC promoter was used to control the expression of $\operatorname{cas} 9$. The recovered cells were plated on MacConkey or LB agar plates containing the appropriate antibiotics. When the araC promoter was used to control Cas9 expression, the inducer (arabinose) was not added in the plates. For the experiment with M9 medium, the recovery was performed in M9 medium. For the stationary phase experiments, the cells were grown in LB until an $\mathrm{OD}_{600}$ of 2.8-3 was reached. In order to control for the cell count, we normalized the $\mathrm{OD}_{600}$ of the cells to mid-log phase $\mathrm{OD}_{600} \mathrm{pf} \sim 0.35-0.4$ and then incubated the cells at $42{ }^{\circ} \mathrm{C}$ to induce lambda Red recombination proteins followed by washing as described with the regular protocol. With the stationary phase cells, two extra wash steps with water to better remove excess salts were performed for preparation of competent cells.

\section{Curing of plasmids with a temperature-sensitive origin of replication}

gRNAs used for insertions, deletions and for tests of gene repression were cloned into editing plasmids with a temperature-sensitive pSC101 origin in a backbone with a carbenicillin resistance marker 
(Phillips et al, 1999). For initial editing/repression, the cells with the temperature-sensitive plasmids were grown at $30{ }^{\circ} \mathrm{C}$. To initiate curing, these plasmids were grown at $37{ }^{\circ} \mathrm{C}$ overnight, then diluted 100 -fold into fresh medium and grown until late-exponential phase. Apart from the test for repression of gene expression, we removed gRNA plasmids used for gene insertions and deletions to avoid potential off-target effects before carrying out subsequent gene editing with different cassettes. pSim5, which also has a temperature-sensitive origin of replication, was also cured from the strains by this procedure. Consequently, it was necessary to re-introduce the pSim5 plasmid into cells for subsequent genome editing.

\section{Calculation of transformation efficiency, apparent editing efficiency and number of recombinants}

In order to measure CFUs per transformation, we plated several dilutions of cells onto LB plates after three hours of recovery at $30^{\circ} \mathrm{C}$ after transformation. Colonies were counted on plates with the most well-resolved colonies and CFUs per transformation were calculated as the number of colony forming units (CFUs) per $\mu \mathrm{g}$ DNA per transformation.

Apparent editing efficiencies were calculated as the percentage of white colonies based upon the numbers of red and white colonies on MacConkey agar plates. MacConkey agar plates were prepared as per the manufacturer's directions with $2 \%$ galactose as the sugar. After $3 \mathrm{~h}$ recovery post-recombineering, several dilutions of the recovery mixture were spread on MacConkey agar plates using glass beads. Colonies were counted on plates with 250-500 resolved colonies to enable calculations of statistical significance. The percentage of white colonies was calculated as the ratio of white to total colonies.

The actual editing efficiencies for Fig 1 were calculated by Sanger sequencing galK from single colonies and subsequently from deep sequencing data as described in the next section. The number of edited was calculated as a product of transformation efficiency and actual editing efficiency.

\section{Genomic deep sequencing}

Cells for genome sequencing were scraped from LB agar plates with 1X PBS after overnight growth of the recovery mixture. Care was taken to collect at least 250-500 colonies for adequate representation of 
the population. Fifty $\mu \mathrm{L}$ of the scraped cell suspension was subjected to centrifugation in a $1.5 \mathrm{~mL}$ tube. The cells were washed twice with PBS and as much of the supernatant was removed as possible. Finally, the cells were suspended in $50 \mu \mathrm{L}$ TE buffer $\left(\mathrm{pH}\right.$ 8.0). The resuspended cells were boiled at $100{ }^{\circ} \mathrm{C}$ for 10 minutes. The genomic region to be sequenced was amplified using primers with Illumina Nextera adapters (Appendix Supplementary Table 2). Amplification was performed with 2X OneTaq polymerase with standard conditions using $25 \mu \mathrm{L}$ of the $2 \mathrm{X}$ OneTaq mastermix, $2.5 \mu \mathrm{L}$ of each primer, $1 \mu \mathrm{L}$ of the boiled cell sample and $19 \mu \mathrm{L}$ of double distilled water. PCR was performed with standard conditions $\left(94{ }^{\circ} \mathrm{C}\right.$ for 10 min, 34 cycles of $94^{\circ} \mathrm{C}$ for $30 \mathrm{~s}, 54^{\circ} \mathrm{C}$ for $30 \mathrm{~s}, 68^{\circ} \mathrm{C}$ for $60 \mathrm{~s}$ and one cycle at $72^{\circ} \mathrm{C}$ for 5 min followed by a hold at $12^{\circ} \mathrm{C}$ ). Each cassette-experiment PCR product was then amplified with a unique experimental Nextera barcode. All sequencing was performed using the Nextera Next-generation sequencing kit with Illumina.

An analysis pipeline was developed for estimating the number of reads with the targeted genomic edit (Supplementary Code 1). The initial assembly of forward and reverse reads was performed using the PandaSeq tool ${ }^{54}$ and the USEARCH algorithm ${ }^{55}$. Experimental barcodes were split using our analysis code (Supplementary Code 1). Reads were mapped to their mutant genotypes using the USEARCH algorithm ${ }^{55}$ with correctly mapped reads having $>98.3 \%$ identity to the target sequence, allowing an error of three mismatches over a $\sim 450$ sequenced region. The actual editing efficiencies were calculated as the ratio of read counts mapped to the mutant phenotype to the total number of reads obtained for the sample. In order to map base change frequencies, the reads were compared to the wild-type sequence with a $95 \%$ identity threshold using the USEARCH algorithm ${ }^{55}$. We developed code to use the identity/mismatch mapping to calculate the frequency of mismatches at each position (Supplementary Code 1). The base change frequency was the ratio of the total number of reads with the base changed to the total number of reads with perfect (100\%) identity to wild-type. Scipy and Numpy kits from python were used for all data analysis.

\section{Supporting Information}

Supplementary Figures 1-17 
Supplementary Table 1 and 2

\section{Acknowledgments}

We would like to acknowledge the University of Colorado Boulder's next-generation sequencing core headed by Dr Amber S. Scott for support with deep sequencing for our study. This work was supported by the US Department of Energy DE-SC0018368 and Inscripta, Inc. During the work, AC was also funded by the 2017-2018 STEM Chateaubriand Fellowship sponsored by the French institut national de la santé et la recherche médicale (INSERM) and S.D.C and A.M. were funded by NIH/NIGMS 5R01 GM124365.

\section{Author Contributions}

Conceptualization: A.C. and R.T.G. Investigation: A.C. and R.G.F. Formal Analysis: A.C., R.G.F., and J.L.K. Visualization: A.C., Resources: A.B.M, M.C.B., E.J.O. and S.D.C. Writing - Original Draft: A.C., R.G.F., E.F.F., S.D.C., and R.T.G. Writing -Review and Editing: A.C., R.T.G., S.D.C., R.G.F, E.F.F., and J.L.K. Supervision: R.T.G. and J.L.K. Funding Acquisition: R.T.G.

\section{Conflict of Interests}

The authors declare conflicting financial interests. R.T.G. has financial interests in the company Inscripta, Inc., which is commercializing the high-throughput CRISPR-Cas9 recombineering-mediated editing technology (CREATE), the cassette design for which has been used in this manuscript.

\section{References}

(1) Jinek, M.; Chylinski, K.; Fonfara, I.; Hauer, M.; Doudna, J. A.; Charpentier, E. A Programmable Dual-RNA-Guided DNA Endonuclease in Adaptive Bacterial Immunity. Science 2012, 1225829.

(2) Jiang, W.; Bikard, D.; Cox, D.; Zhang, F.; Marraffini, L. A. RNA-Guided Editing of Bacterial Genomes Using CRISPR-Cas Systems. Nat. Biotechnol. 2013, 31 (3), 233-239.

(3) Bikard, D.; Hatoum-Aslan, A.; Mucida, D.; Marraffini, L. A. CRISPR Interference Can Prevent Natural Transformation and Virulence Acquisition during in Vivo Bacterial Infection. Cell Host Microbe 2012, 12 (2), 177-186.

(4) Jiang, Y.; Chen, B.; Duan, C.; Sun, B.; Yang, J.; Yang, S. Multigene Editing in the Escherichia Coli Genome via the CRISPR-Cas9 System. Appl. Environ. Microbiol. 2015, 81 (7), 2506-2514.

(5) Garst, A. D.; Bassalo, M. C.; Pines, G.; Lynch, S. A.; Halweg-Edwards, A. L.; Liu, R.; Liang, L.; 
Wang, Z.; Zeitoun, R.; Alexander, W. G.; et al. Genome-Wide Mapping of Mutations at SingleNucleotide Resolution for Protein, Metabolic and Genome Engineering. Nat. Biotechnol. 2017, 35 (1), 48-55.

(6) Liu, R.; Liang, L.; Choudhury, A.; Bassalo, M. C.; Garst, A. D.; Tarasava, K.; Gill, R. T. Iterative Genome Editing of Escherichia Coli for 3-Hydroxypropionic Acid Production. Metab. Eng. 2018, 47, 303-313.

(7) Liu, R.; Liang, L.; Garst, A. D.; Choudhury, A.; Nogué, V. S. I.; Beckham, G. T.; Gill, R. T. Directed Combinatorial Mutagenesis of Escherichia Coli for Complex Phenotype Engineering. Metab. Eng. 2018, 47, 10-20.

(8) Liang, L.; Liu, R.; Garst, A. D.; Lee, T.; Nogué, V. S. I.; Beckham, G. T.; Gill, R. T. CRISPR EnAbled Trackable Genome Engineering for Isopropanol Production in Escherichia Coli. Metab. Eng. 2017, 41, 1-10.

(9) Bassalo, M. C.; Garst, A. D.; Choudhury, A.; Grau, W. C.; Oh, E. J.; Spindler, E.; Lipscomb, T.; Gill, R. T. Deep Scanning Lysine Metabolism in Escherichia Coli. Mol. Syst. Biol. 2018, 14 (11), e8371.

(10) Zerbini, F.; Zanella, I.; Fraccascia, D.; König, E.; Irene, C.; Frattini, L. F.; Tomasi, M.; Fantappiè, L.; Ganfini, L.; Caproni, E.; et al. Large Scale Validation of an Efficient CRISPR/Cas-Based Multi Gene Editing Protocol in Escherichia Coli. Microb. Cell Fact. 2017, 16 (1), 68.

(11) Cui, L.; Bikard, D. Consequences of Cas9 Cleavage in the Chromosome of Escherichia Coli. Nucleic Acids Res. 2016, 44 (9), 4243-4251.

(12) Moreb, E. A.; Hoover, B.; Yaseen, A.; Valyasevi, N.; Roecker, Z.; Menacho-Melgar, R.; Lynch, M. D. Managing the SOS Response for Enhanced CRISPR-Cas-Based Recombineering in E. Coli through Transient Inhibition of Host RecA Activity. ACS Synth. Biol. 2017, 6 (12), 2209-2218.

(13) Wilson, L. O. W.; O'Brien, A. R.; Bauer, D. C. The Current State and Future of CRISPR-Cas9 gRNA Design Tools. Front. Pharmacol. 2018, 9, 749.

(14) Guo, J.; Wang, T.; Guan, C.; Liu, B.; Luo, C.; Xie, Z.; Zhang, C.; Xing, X.-H. Improved sgRNA Design in Bacteria via Genome-Wide Activity Profiling. Nucleic Acids Res. 2018, 46 (14), 70527069.

(15) Doench, J. G.; Hartenian, E.; Graham, D. B.; Tothova, Z.; Hegde, M.; Smith, I.; Sullender, M.; Ebert, B. L.; Xavier, R. J.; Root, D. E. Rational Design of Highly Active sgRNAs for CRISPR-Cas9Mediated Gene Inactivation. Nat. Biotechnol. 2014, 32 (12), 1262-1267.

(16) Farasat, I.; Salis, H. M. A Biophysical Model of CRISPR/Cas9 Activity for Rational Design of Genome Editing and Gene Regulation. PLoS Comput. Biol. 2016, 12 (1), e1004724.

(17) Jiang, F.; Doudna, J. A. CRISPR-Cas9 Structures and Mechanisms. Annu. Rev. Biophys. 2017, 46, 505-529.

(18) Thaler, D. S.; Stahl, M. M.; Stahl, F. W. Double-Chain-Cut Sites Are Recombination Hotspots in the Red Pathway of Phage Lambda. J. Mol. Biol. 1987, 195 (1), 75-87.

(19) Pósfai, G.; Kolisnychenko, V.; Bereczki, Z.; Blattner, F. R. Markerless Gene Replacement in Escherichia Coli Stimulated by a Double-Strand Break in the Chromosome. Nucleic Acids Res. 1999, 27 (22), 4409-4415.

(20) Poteete, A. R.; Fenton, A. C. Efficient Double-Strand Break-Stimulated Recombination Promoted by the General Recombination Systems of Phages Lambda and P22. Genetics 1993, 134 (4), 1013 1021.

(21) Sawitzke, J. A.; Costantino, N.; Li, X.-T.; Thomason, L. C.; Bubunenko, M.; Court, C.; Court, D. L. Probing Cellular Processes with Oligo-Mediated Recombination and Using the Knowledge Gained to Optimize Recombineering. J. Mol. Biol. 2011, 407 (1), 45-59.

(22) Taniguchi, Y.; Choi, P. J.; Li, G.-W.; Chen, H.; Babu, M.; Hearn, J.; Emili, A.; Sunney Xie, X. Quantifying E. Coli Proteome and Transcriptome with Single-Molecule Sensitivity in Single Cells. Science 2010, 329 (5991), 533-538.

(23) Reynolds, T. S.; Gill, R. T. Quantifying Impact of Chromosome Copy Number on Recombination in Escherichia Coli. ACS Synth. Biol. 2015, 4 (7), 776-780. 
(24) Jones, D. L.; Leroy, P.; Unoson, C.; Fange, D.; Ćurić, V.; Lawson, M. J.; Elf, J. Kinetics of dCas9 Target Search in Escherichia Coli. Science 2017, 357 (6358), 1420-1424.

(25) Davis, J. H.; Rubin, A. J.; Sauer, R. T. Design, Construction and Characterization of a Set of Insulated Bacterial Promoters. Nucleic Acids Res. 2011, 39 (3), 1131-1141.

(26) Chayot, R.; Montagne, B.; Mazel, D.; Ricchetti, M. An End-Joining Repair Mechanism in Escherichia Coli. Proc. Natl. Acad. Sci. U. S. A. 2010, 107 (5), 2141-2146.

(27) Li, Y.; Lin, Z.; Huang, C.; Zhang, Y.; Wang, Z.; Tang, Y.-J.; Chen, T.; Zhao, X. Metabolic Engineering of Escherichia Coli Using CRISPR-Cas9 Meditated Genome Editing. Metab. Eng. 2015, 31, 13-21.

(28) Costantino, N.; Court, D. L. Enhanced Levels of Lambda Red-Mediated Recombinants in Mismatch Repair Mutants. Proc. Natl. Acad. Sci. U. S. A. 2003, 100 (26), 15748-15753.

(29) Cui, L.; Vigouroux, A.; Rousset, F.; Varet, H.; Khanna, V.; Bikard, D. A CRISPRi Screen in E. Coli Reveals Sequence-Specific Toxicity of dCas9. Nat. Commun. 2018, 9 (1), 1912.

(30) Hsu, P. D.; Scott, D. A.; Weinstein, J. A.; Ran, F. A.; Konermann, S.; Agarwala, V.; Li, Y.; Fine, E. J.; Wu, X.; Shalem, O.; et al. DNA Targeting Specificity of RNA-Guided Cas9 Nucleases. Nat. Biotechnol. 2013, 31 (9), 827-832.

(31) Kreuzer, K. N. DNA Damage Responses in Prokaryotes: Regulating Gene Expression, Modulating Growth Patterns, and Manipulating Replication Forks. Cold Spring Harb. Perspect. Biol. 2013, 5 (11), a012674.

(32) Mallik, S.; Popodi, E. M.; Hanson, A. J.; Foster, P. L. Interactions and Localization of Escherichia Coli Error-Prone DNA Polymerase IV after DNA Damage. J. Bacteriol. 2015, 197 (17), 2792-2809.

(33) Murphy, K. C. The Lambda Gam Protein Inhibits RecBCD Binding to dsDNA Ends. J. Mol. Biol. 2007, 371 (1), 19-24.

(34) Gong, S.; Yu, H. H.; Johnson, K. A.; Taylor, D. W. DNA Unwinding Is the Primary Determinant of CRISPR-Cas9 Activity. Cell Rep. 2018, 22 (2), 359-371.

(35) Thyme, S. B.; Akhmetova, L.; Montague, T. G.; Valen, E.; Schier, A. F. Internal Guide RNA Interactions Interfere with Cas9-Mediated Cleavage. Nat. Commun. 2016, 7, 11750.

(36) Phillips, G. J. New Cloning Vectors with Temperature-Sensitive Replication. Plasmid 1999, 41 (1), 78-81.

(37) Qi, L. S.; Larson, M. H.; Gilbert, L. A.; Doudna, J. A.; Weissman, J. S.; Arkin, A. P.; Lim, W. A. Repurposing CRISPR as an RNA-Guided Platform for Sequence-Specific Control of Gene Expression. Cell 2013, 152 (5), 1173-1183.

(38) Haeussler, M.; Schönig, K.; Eckert, H.; Eschstruth, A.; Mianné, J.; Renaud, J.-B.; SchneiderMaunoury, S.; Shkumatava, A.; Teboul, L.; Kent, J.; et al. Evaluation of off-Target and on-Target Scoring Algorithms and Integration into the Guide RNA Selection Tool CRISPOR. Genome Biol. 2016, 17 (1), 148.

(39) Singh, D.; Sternberg, S. H.; Fei, J.; Doudna, J. A.; Ha, T. Real-Time Observation of DNA Recognition and Rejection by the RNA-Guided Endonuclease Cas9. Nat. Commun. 2016, 7, 12778.

(40) Boyle, E. A.; Andreasson, J. O. L.; Chircus, L. M.; Sternberg, S. H.; Wu, M. J.; Guegler, C. K.; Doudna, J. A.; Greenleaf, W. J. High-Throughput Biochemical Profiling Reveals Sequence Determinants of dCas9 off-Target Binding and Unbinding. Proc. Natl. Acad. Sci. U. S. A. 2017, 114 (21), 5461-5466.

(41) Kiani, S.; Chavez, A.; Tuttle, M.; Hall, R. N.; Chari, R.; Ter-Ovanesyan, D.; Qian, J.; Pruitt, B. W.; Beal, J.; Vora, S.; et al. Cas9 gRNA Engineering for Genome Editing, Activation and Repression. Nat. Methods 2015, 12 (11), 1051-1054.

(42) Mekler, V.; Kuznedelov, K.; Minakhin, L.; Murugan, K.; Sashital, D. G.; Severinov, K. CRISPRCas Molecular Beacons as Tool for Studies of Assembly of CRISPR-Cas Effector Complexes and Their Interactions with DNA. Methods Enzymol. 2019, 616, 337-363.

(43) Mekler, V.; Minakhin, L.; Severinov, K. Mechanism of Duplex DNA Destabilization by RNAGuided Cas9 Nuclease during Target Interrogation. Proc. Natl. Acad. Sci. U. S. A. 2017, 114 (21), 5443-5448. 
(A) Editing cassettes consist of a homology repair template (HRT) that introduces a targeted mutation and a synonymous PAM mutation (SPM) at the genomic target. The SPM selects for editing events by replacing the NGG PAM to prevent subsequent Cas9:gRNA mediated DSB-induced cell death.

(B) Correlation between Cas9:gRNA-induced cell death and editing editing efficiency using 35 unique gRNAs targeting either galK (black) or other genes (grey). We categorized the 15 gRNAs above this threshold into Group 1. Nine gRNAs that caused negligible Cas9:gRNA-induced cell death in 
the absence of a HRT (with a p-value $>0.05$ compared to a non-targeting gRNA) were categorized into Group 3. The remaining gRNAs were categorized into Group 2.

(C) Number of total CFUs (CFUs/( $\mu \mathrm{g}$ DNA*mL transformation)), edited CFUs, and editing efficiency (\%) (top text) after transformation of plasmid carrying a galK-targeting cassette from each gRNA group into Cas9-expressing cells. The non-targeting gRNA was expressed with the HRT used for the Group 1 cassette. The uncertainties in the top text represent the uncertainties in the measurement of editing efficiency, the error bars for the black bars represent the uncertainties in the measurement of CFUs per transformation, and the error bars for the green bars represent the uncertainties in the measurement number of edited CFUs.

All values represent mean +/- s.d., $n=3$.

Figure 2. Variation in the percentage of white colonies with target genomic position and cas9 expression

(A) (left) Design of an editing cassette for the introduction of a stop codon (red) and a synonymous PAM mutation (blue) in galK; (right) a MacConkey agar plate with white (edited) and red (unedited) colonies. The percentage of white colonies is calculated as the ratio of white to total colonies.

(B) Locations at which galK was integrated into the genome, annotated by distances from the terminus of replication (ter).

(C) The percentage of white colonies for different cassettes with the target galK gene integrated at different genomic loci.

(D) The percentage of white colonies for different cassettes with increasing cas 9 promoter strength.

All values represent mean $+/-$ s.d., $n=3$.

Figure 3. Actual editing behavior for Group 1 cassettes 


\section{Figure 4. Editing behavior for Group 2 cassettes}

(A) Number of total and edited CFUs for galK integrated at different genomic positions (left) and using different $\operatorname{cas} 9$ promoters (right) for Group 2 Cassette 4 . The editing efficiency is shown above the graph for each position and promoter. The uncertainties in the top text represent the uncertainties in the measurement of editing efficiency, the error bars for the black bars represent the uncertainties 
in the measurement of CFUs per transformation, and the error bars for the green bars represent the uncertainties in the measurement number of edited CFUs.

(B) Number of total and edited CFUs for galK integrated at different genomic positions (left) and using different cas 9 promoters (right) for Group 2 Cassette 5. The editing efficiency is shown above the graph for each position and promoter. The uncertainties in the top text represent the uncertainties in the measurement of editing efficiency, the error bars for the black bars represent the uncertainties in the measurement of CFUs per transformation, and the error bars for the green bars represent the uncertainties in the measurement number of edited CFUs.

(C) Base change frequencies at the target site for Cassette 4. Positions with a significant number of non-targeted mutations $(\mathrm{P}$-value $<0.0001)$ are indicated with black arrows.

(D) Base change frequencies at the target site upon transforming a plasmid carrying only the gRNA of editing Cassette 5 (i.e, with no HRT) after induction of lambda Red enzymes.

Number of CFUs represents mean +/- s.d., $n=3$. editing efficiency values represent mean $+/$ - s.d., $n=2$.

\section{Figure 5. Editing behavior for Group 3 cassettes}

(A) Comparison of actual editing efficiency and the percentage of white colonies for galK integrated at different genomic positions and promoters achieved with Group 3 editing cassettes 6,7 and 8 .

(B) Comparison of loss of galK function using the MacConkey agar screen with Group 3 gRNAs expressed on a temperature curable plasmid at $30^{\circ} \mathrm{C}$ (retained) and $37{ }^{\circ} \mathrm{C}$ (cured). The plates on the far right demonstrate an edited control grown at $37^{\circ} \mathrm{C}$ in which cells were edited using a Group 1 gRNA and retained the edit after curing the editing plasmids from the cell.

editing efficiency values represent mean $+/-$ s.d., $n=2$. 


\section{Figure 6. The effect of mutations in the gRNA on editing behavior}

(A) Number of total and edited CFUs, and editing efficiency for Cassette 1 variants using two different cas 9 promoters. Mutations were introduced into Group 1 Cassette 1 gRNA in bases 4, 8 and 12 proximal to the PAM. The editing efficiency is shown above the graph for each position and promoter. The uncertainties in the top text represent the uncertainties in the measurement of editing efficiency, the error bars for the black bars represent the uncertainties in the measurement of CFUs per transformation, and the error bars for the green bars represent the uncertainties in the measurement number of edited CFUs.

(B) Comparison of actual editing efficiency and the percentage of white colonies for Group 1 Cassette 2 variants with two different $\operatorname{cas} 9$ promoters.

(C) Base change frequencies at the target site observed after editing with Cassette 1 with a mutation in the gRNA at PAM proximal base 4. Positions with significant non-targeted mutations are indicated with black arrows.

Number of CFUs represents mean +/- s.d., $n=3$. editing efficiency values represent mean $+/$ - s.d., $n=2$.

Figure 7: Editing with changes in growth and in multiplex

(A) Number of CFUs, number of edited cells and editing efficiency for gRNAs from Groups 1, 2 and 3 when cells were recovered in either LB medium (LB) or M9 minimal medium (M9), and when editing was carried out in exponential phase (exp) or stationary phase (stat). The editing efficiency is shown above the graph for each position and promoter. The uncertainties in the top text represent the uncertainties in the measurement of editing efficiency, the error bars for the black bars represent the uncertainties in the measurement of CFUs per transformation, and the error bars for the green bars represent the uncertainties in the measurement number of edited CFUs.

(B) Number of CFUs, edited cells and editing efficiency with a mixture of four cassettes when cells were recovered in LB medium (LB) in exponential (exp) or stationary phase (stat). The editing 
efficiency is shown above the graph for each position and promoter. The uncertainties in the top text represent the uncertainties in the measurement of editing efficiency, the error bars for the black bars represent the uncertainties in the measurement of CFUs per transformation, and the error bars for the green bars represent the uncertainties in the measurement number of edited CFUs.

Number of CFUs represents mean $+/-$ s.d., $n=3$. editing efficiency values represent mean $+/$ - s.d., $n=2$. 
B.

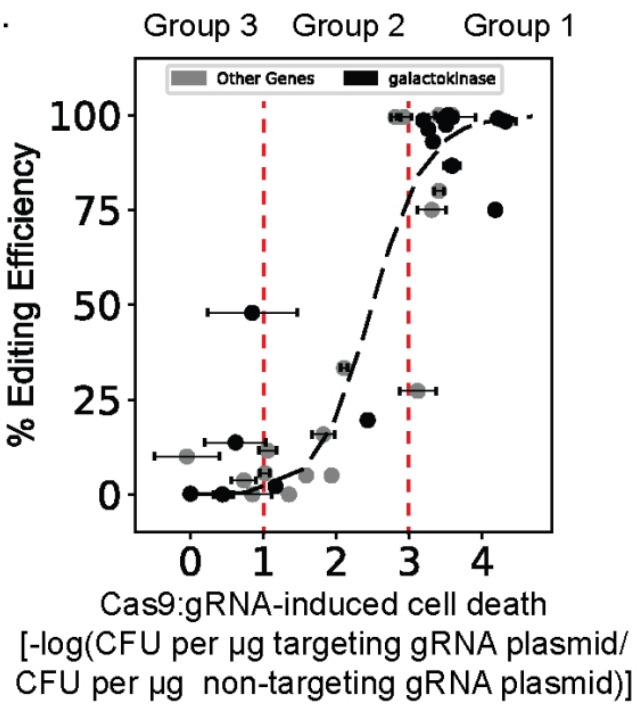

\section{C.}

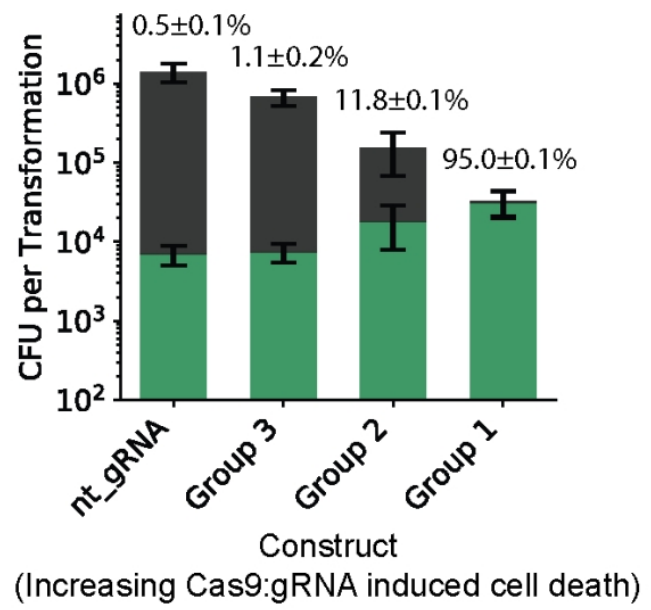

Figure 1. Editing is driven by Cas9:gRNA-induced cell death but with unpredictable deviations

(A) Editing cassettes consist of a homology repair template (HRT) that introduces a targeted mutation and a synonymous PAM mutation (SPM) at the genomic target. The SPM selects for editing events by replacing the NGG PAM to prevent subsequent Cas9:gRNA mediated DSB-induced cell death.

(B) Correlation between Cas9:gRNA-induced cell death and editing editing efficiency using 35 unique gRNAs targeting either galK (black) or other genes (grey). We categorized the 15 gRNAs above this threshold into Group 1. Nine gRNAs that caused negligible Cas9:gRNA-induced cell death in the absence of a HRT (with a p-value $>0.05$ compared to a non-targeting gRNA) were categorized into Group 3. The remaining gRNAs were categorized into Group 2.

(C) Number of total CFUs (CFUs/( $\gg \mathrm{DNA} * \mathrm{~mL}$ transformation)), edited CFUs, and editing efficiency (\%) (top text) after transformation of plasmid carrying a galK-targeting cassette from each gRNA group into Cas9-expressing cells. The non-targeting gRNA was expressed with the HRT used for the Group 1 cassette. The uncertainties in the top text represent the uncertainties in the measurement of editing efficiency, the error bars for the black bars represent the uncertainties in the measurement of CFUs per transformation, and the error bars for the green bars represent the uncertainties in the measurement number of edited 
CFUs.

All values represent mean +/- s.d., $n=3$. 
A.

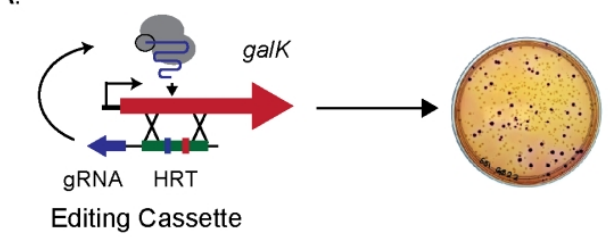

B.

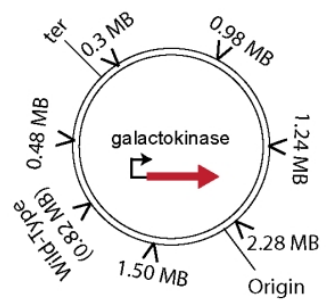

Group 1
Editing Cassette $0 \quad$ Editing Cassette 1

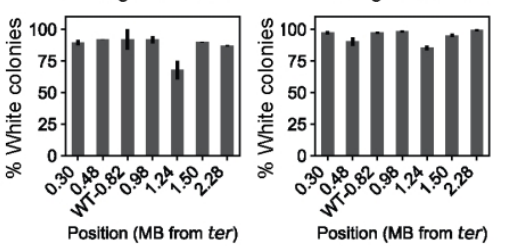

Editing Cassette $2 \quad$ Position (MB rom ter)
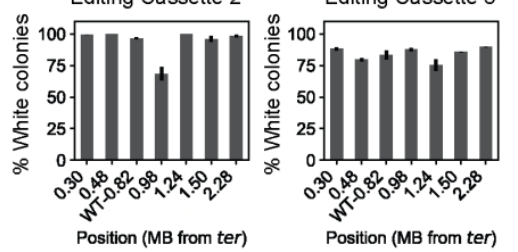

D.

Group 1
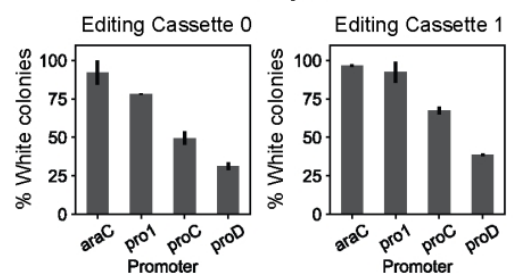

Editing Cassette 2
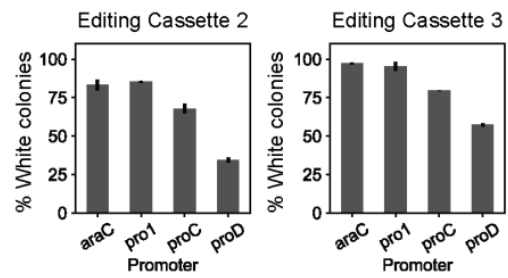

Group 2

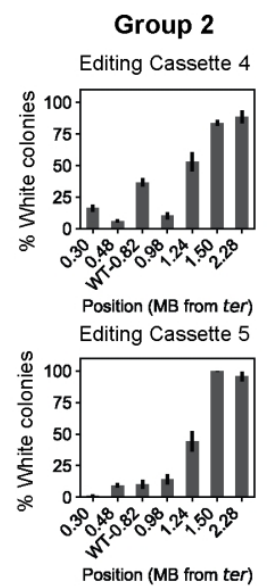

Group 2

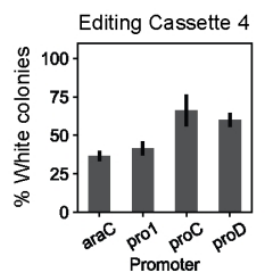

Editing Cassette 5

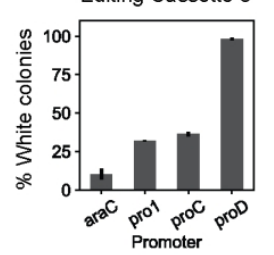

Group 3
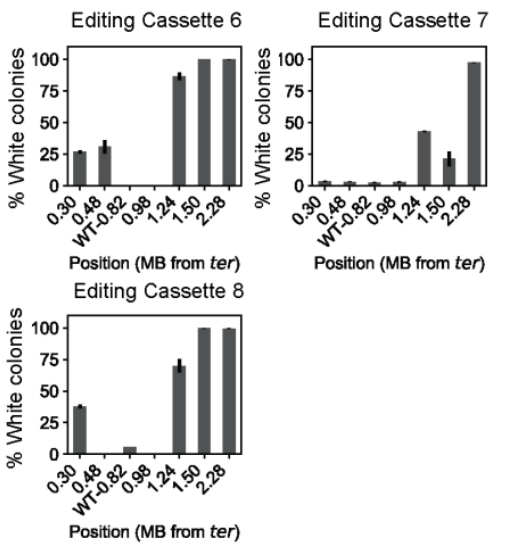

Group 3

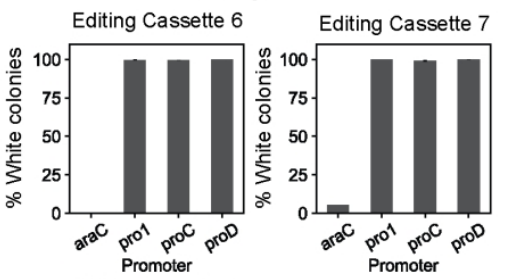

Editing Cassette 8

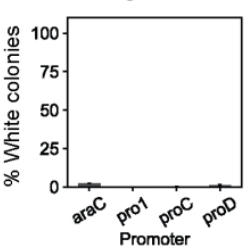

Figure 2. Variation in the percentage of white colonies with target genomic position and cas9 expression

(A) (left) Design of an editing cassette for the introduction of a stop codon (red) and a synonymous PAM mutation (blue) in galk; (right) a MacConkey agar plate with white (edited) and red (unedited) colonies. The percentage of white colonies is calculated as the ratio of white to total colonies.

(B) Locations at which galK was integrated into the genome, annotated by distances from the terminus of replication (ter).

(C) The percentage of white colonies for different cassettes with the target galK gene integrated at different genomic loci.

(D) The percentage of white colonies for different cassettes with increasing cas9 promoter strength.

All values represent mean +/- s.d., $n=3$. 
A.
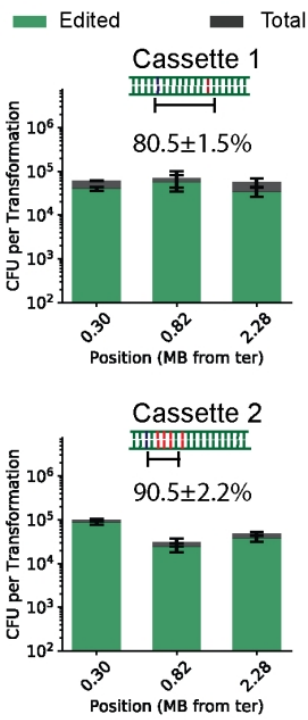

C.

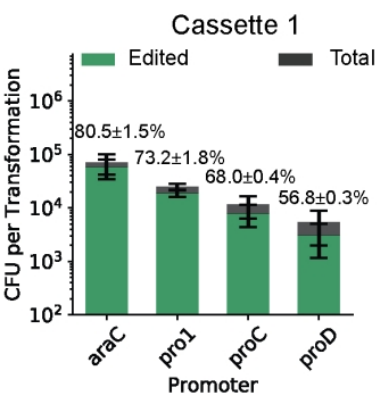

D.

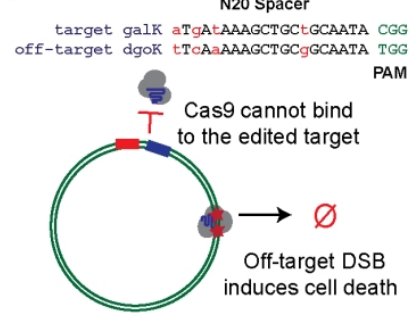

B.

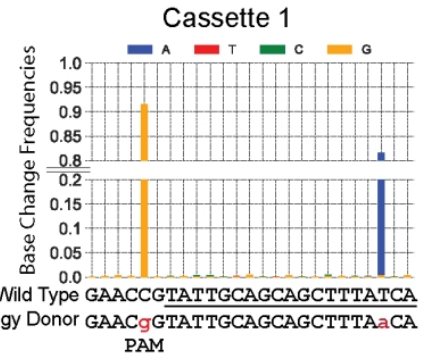

AM

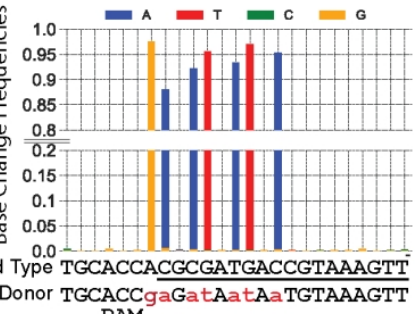

PAM

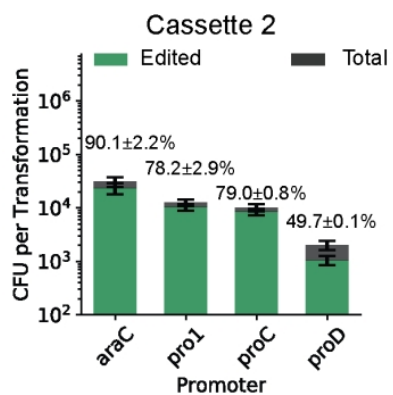

E.

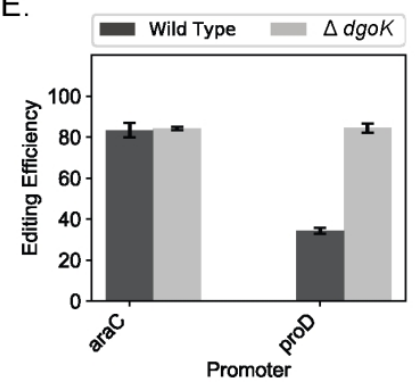

Figure 3. Actual editing behavior for Group 1 cassettes

(A) Total and edited CFUs after editing of galK integrated at different target positions with Group 1 editing cassettes. The HRT design for each cassette is above the graph. The uncertainties in the top text represent the uncertainties in the measurement of editing efficiency, the error bars for the black bars represent the uncertainties in the measurement of CFUs per transformation, and the error bars for the green bars represent the uncertainties in the measurement number of edited CFUs.

(B) Base-change frequency at target sites for Group 1 editing cassettes. The target sequence and sequence after recombination are shown on the x-axis. The PAM is indicated by the text, the N20 spacer sequence is underlined, and the bases targeted for mutation are red and lowercase.

(C) Number of total and edited CFUs achieved for Group 1 editing cassettes targeting galK at the wild-type locus when cas9 expression is controlled by different promoters. The uncertainties in the top text represent the uncertainties in the measurement of editing efficiency, the error bars for the black bars represent the uncertainties in the measurement of CFUs per transformation, and the error bars for the green bars 
represent the uncertainties in the measurement number of edited CFUs.

(D) The Cassette 1 gRNA target site in galK and major off-target site in dgoK. Differences between the ontarget and off-target spacer sequences are shown in red and lowercase.

(E) Comparison of actual editing efficiency between wild-type and $\Delta$ dgoK strains with two different cas 9 promoters.

Number of CFUs represents mean +/- s.d., $n=3$. editing efficiency values represent mean $+/-$ s.d., $n=2$. 
A.

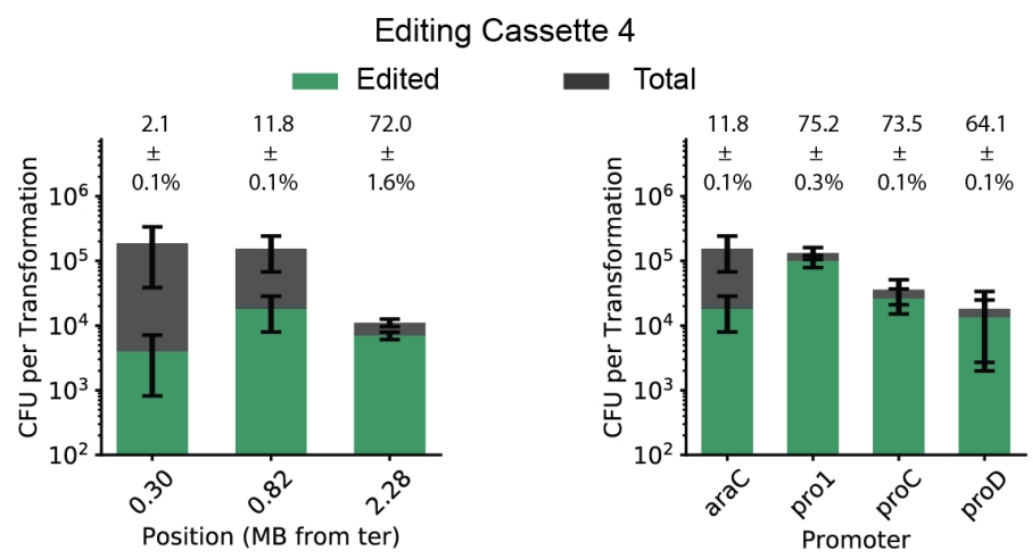

B.

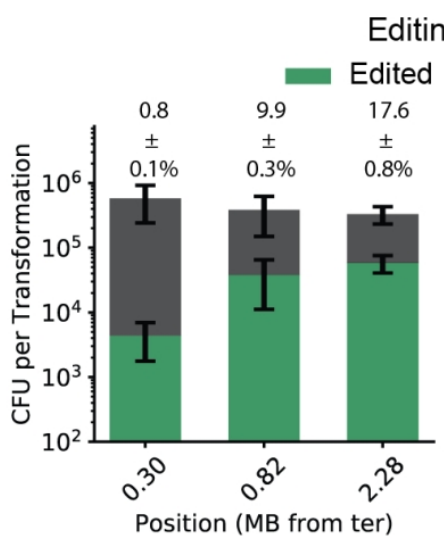

Editing Cassette 5

C.

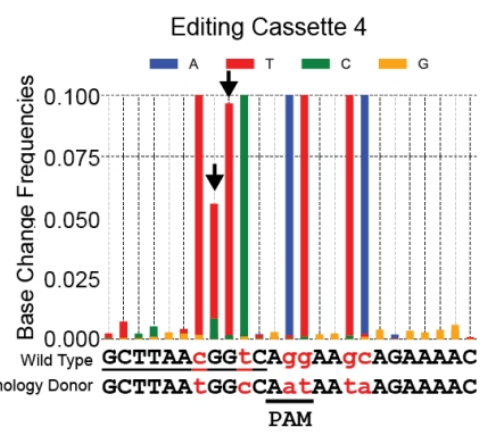

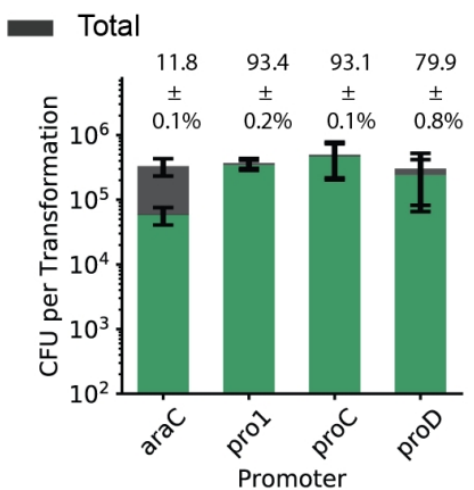

D.

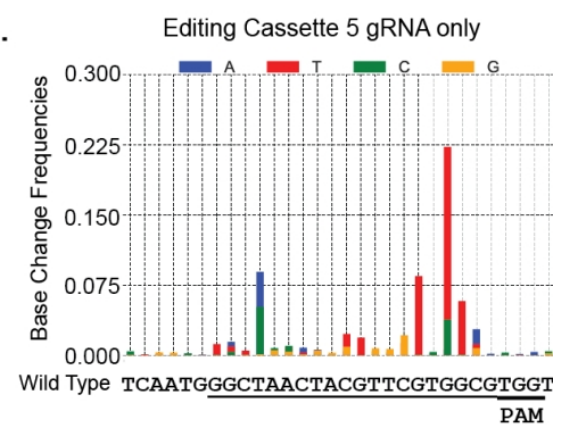

Figure 4. Editing behavior for Group 2 cassettes

(A) Number of total and edited CFUs for galK integrated at different genomic positions (left) and using different cas9 promoters (right) for Group 2 Cassette 4 . The editing efficiency is shown above the graph for each position and promoter. The uncertainties in the top text represent the uncertainties in the

measurement of editing efficiency, the error bars for the black bars represent the uncertainties in the measurement of CFUs per transformation, and the error bars for the green bars represent the uncertainties in the measurement number of edited CFUs.

(B) Number of total and edited CFUs for galK integrated at different genomic positions (left) and using different cas9 promoters (right) for Group 2 Cassette 5. The editing efficiency is shown above the graph for each position and promoter. The uncertainties in the top text represent the uncertainties in the

measurement of editing efficiency, the error bars for the black bars represent the uncertainties in the measurement of CFUs per transformation, and the error bars for the green bars represent the uncertainties in the measurement number of edited CFUs. 
(C) Base change frequencies at the target site for Cassette 4. Positions with a significant number of nontargeted mutations (P-value $<0.0001)$ are indicated with black arrows.

(D) Base change frequencies at the target site upon transforming a plasmid carrying only the gRNA of editing Cassette 5 (i.e, with no HRT) after induction of lambda Red enzymes.

Number of CFUs represents mean +/- s.d., $n=3$. editing efficiency values represent mean +/- s.d., $n=2$. 
A.
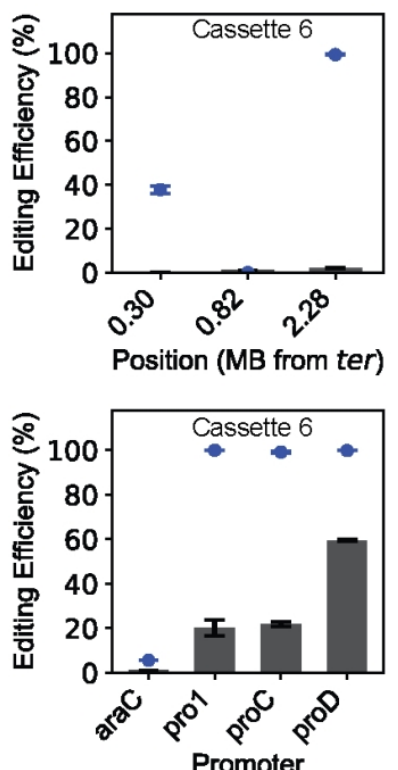

B.

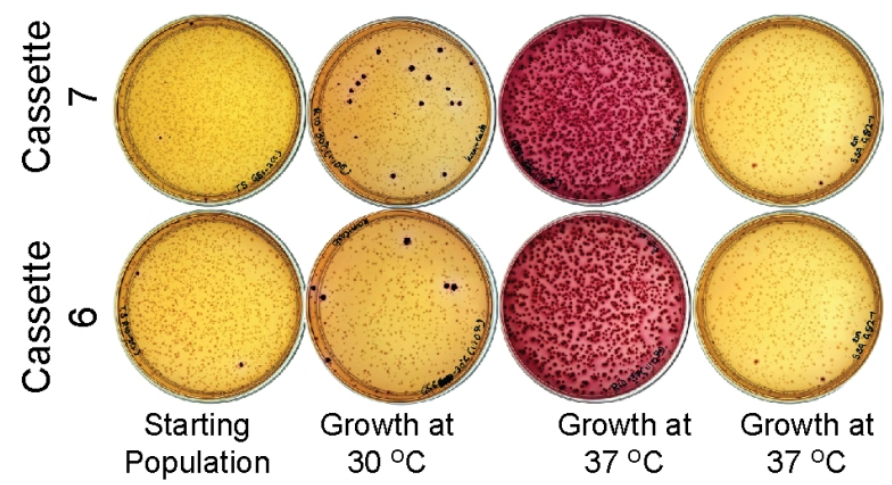

Figure 5. Editing behavior for Group 3 cassettes

(A) Comparison of actual editing efficiency and the percentage of white colonies for galK integrated at different genomic positions and promoters achieved with Group 3 editing cassettes 6, 7 and 8 .

(B) Comparison of loss of galK function using the MacConkey agar screen with Group 3 gRNAs expressed on a temperature curable plasmid at $30 \mathrm{OC}$ (retained) and $37 \mathrm{OC}$ (cured). The plates on the far right demonstrate an edited control grown at $37 \mathrm{OC}$ in which cells were edited using a Group 1 gRNA and retained the edit after curing the editing plasmids from the cell.

editing efficiency values represent mean $+/-$ s.d., $n=2$. 

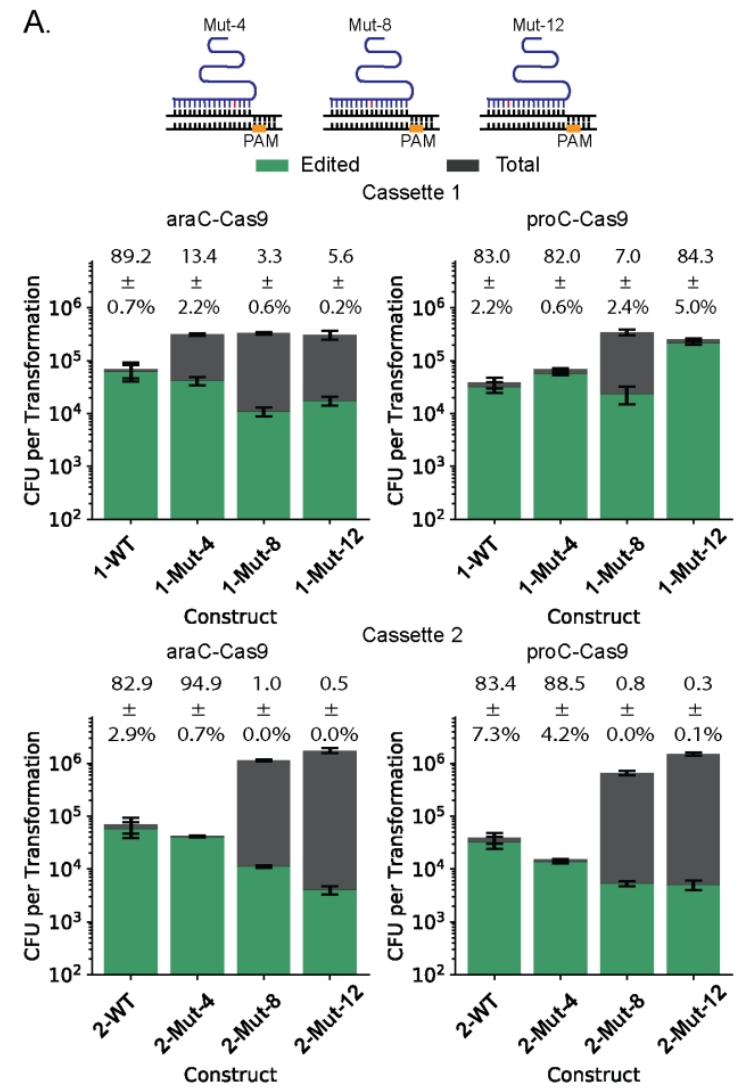

B.

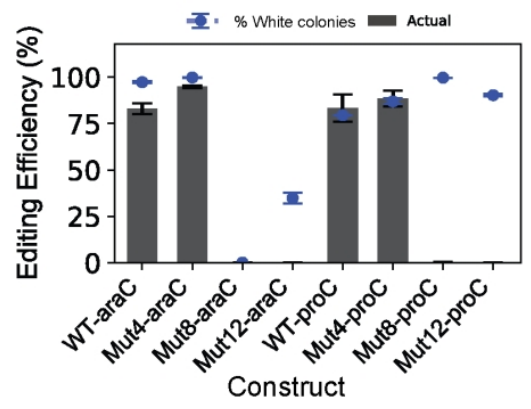

C.

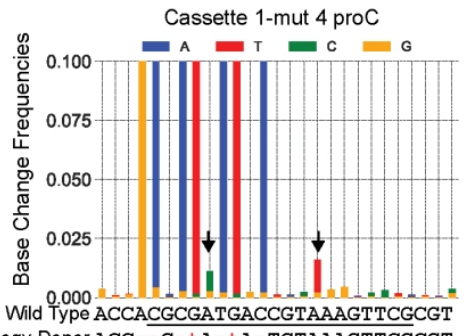

Homology Donor ACCgaGatAa tAaTGTAAAGTTCGCGT PAM

Figure 6 . The effect of mutations in the gRNA on editing behavior

(A) Number of total and edited CFUs, and editing efficiency for Cassette 1 variants using two different cas9 promoters. Mutations were introduced into Group 1 Cassette 1 gRNA in bases 4, 8 and 12 proximal to the PAM. The editing efficiency is shown above the graph for each position and promoter. The uncertainties in the top text represent the uncertainties in the measurement of editing efficiency, the error bars for the black bars represent the uncertainties in the measurement of CFUs per transformation, and the error bars for the green bars represent the uncertainties in the measurement number of edited CFUs.

(B) Comparison of actual editing efficiency and the percentage of white colonies for Group 1 Cassette 2 variants with two different cas9 promoters.

(C) Base change frequencies at the target site observed after editing with Cassette 1 with a mutation in the gRNA at PAM proximal base 4. Positions with significant non-targeted mutations are indicated with black arrows.

Number of CFUs represents mean +/- s.d., $n=3$. editing efficiency values represent mean +/- s.d., $n=2$. 
A.

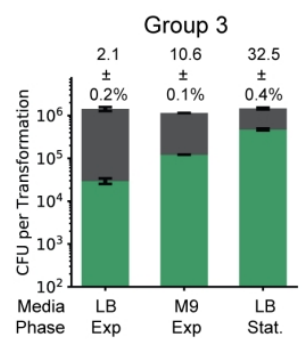

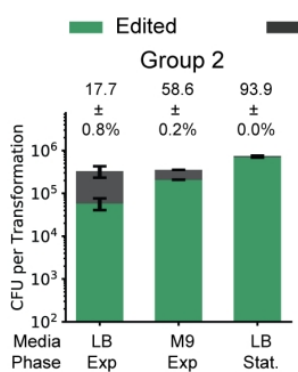

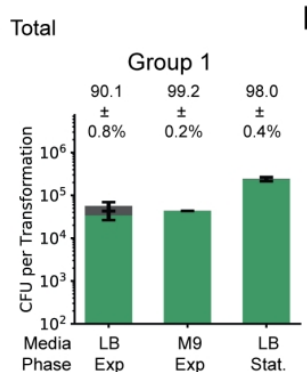

B.

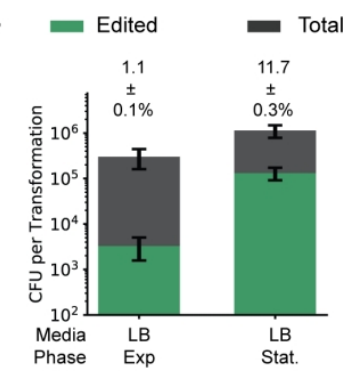

Figure 7: Editing with changes in growth and in multiplex

(A) Number of CFUs, number of edited cells and editing efficiency for gRNAs from Groups 1, 2 and 3 when cells were recovered in either LB medium (LB) or M9 minimal medium (M9), and when editing was carried out in exponential phase (exp) or stationary phase (stat). The editing efficiency is shown above the graph

for each position and promoter. The uncertainties in the top text represent the uncertainties in the measurement of editing efficiency, the error bars for the black bars represent the uncertainties in the measurement of CFUs per transformation, and the error bars for the green bars represent the uncertainties in the measurement number of edited CFUs.

(B) Number of CFUs, edited cells and editing efficiency with a mixture of four cassettes when cells were recovered in LB medium (LB) in exponential (exp) or stationary phase (stat). The editing efficiency is shown above the graph for each position and promoter. The uncertainties in the top text represent the uncertainties in the measurement of editing efficiency, the error bars for the black bars represent the uncertainties in the measurement of CFUs per transformation, and the error bars for the green bars represent the uncertainties in the measurement number of edited CFUs.

Number of CFUs represents mean +/- s.d., $n=3$. editing efficiency values represent mean +/- s.d., $n=2$. 